\title{
螺吡喃分子光开关
}

\author{
张国峰陈涛李冲龚文亮 \\ Matthew P. Aldred 朱明强* \\ (华中科技大学 武汉光电国家实验室(筹) 武汉 430074)
}

\begin{abstract}
摘要 螺吡喃是一类研究最早, 最广泛的有机光致变色分子. 利用光照前后螺吡喃分子结构上和光化学与光物理性质 的变化, 将其作为分子光开关材料被广泛应用于光电器件、化学传感、药物控制释放等领域. 综述了部分螺吡喃分子 光开关的合成和性质及其在相关领域中的应用，对其作为新型的荧光探针未来在生物成像领域的应用做了展望.
\end{abstract}

关键词 螺吡喃; 分子光开关; 生物成像

\section{Spiropyran-Based Molecular Photoswitches}

\author{
Zhang, Guofeng Chen, Tao Li, Chong Gong, Wenliang \\ Aldred, Matthew P. Zhu, Mingqiang* \\ (Wuhan National Laboratory for Optoelectronics, Huazhong University of Science and Technology, Wuhan 430074)
}

\begin{abstract}
Spiropyran is one of the classic photochromic compounds, which undergoes photoisomerization under ultraviolet exposure with its structure transformed from a non-polar, closed and colorless spiro-form to a highly polar, open and colorful cyanine-form with extended $\pi$-electron delocalization. This has encourged researchers to employ this material with unique photochemical and photophysical properties as a molecular photoswitch in the field of optoelectronic devices, chemical biosensing, controlled release drug delivery and so on. In this review, noticeable designs, synthesis, properties of some spiropyran derivatives and their applications in relative fields are summarized. Finally, as a promising fluorescent probe, the future developing prospects in bioimaging are discussed and highlighted.
\end{abstract}

Keywords spiropyran; molecular photoswitches; bioimaging

光致变色现象是指当受到一定波长的光照射时，化 合物 $\mathrm{A}$ 通过异构化反应转变为 $\mathrm{B}$, 而化合物 $\mathrm{B}$ 在另一种 波长的光照射或者热的作用下, 又可以转变恢复为 $\mathrm{A}$. 即 $\mathrm{A}$ 或 $\mathrm{B}$ 作为分子光开关单元, 在受到外界刺激, 如不 同波长光照射下，双态之间可以进行可逆的相互转换. 通常伴随着光开关分子之间可逆光异构化反应的进行, 物质 $\mathrm{A}$ 和物质 $\mathrm{B}$ 的化学结构会发生很大的变化, 而其对 应的光化学性质和光物理性质, 如分子的吸收光谱 ${ }^{[1]}$ 、 荧光光谱、共轭程度、导电性、磁性、电化学性质、配 位能力、极性、折光指数、介电常数和亲疏水性质也会 随之发生较大的变化 ${ }^{[2]}$. 基于其特殊性质, 光致变色材
料在光电器件 ${ }^{[3]}$ 、超高密度信息存储 ${ }^{[4]}$ 、分子逻辑开关 ${ }^{[5]}$ 、 离子识别 ${ }^{[6]}$ 、分子自助装 ${ }^{[7]}$ 、药物控制释放 ${ }^{[8]}$ 、超分辨成 像 ${ }^{[9]}$ 等诸多领域得到了广泛的研究和应用. 目前研究的 光致变色材料(Scheme 1)主要分为：芳香族偶氮化合 物、二芳基乙烯、螺吡喃和螺噁溙、俘精酸酕等几类.

芳香族偶氮类化合物在紫外光的照射下，依靠分子 中偶氮基团的光致顺反异构而产生光致变色现象. 利用 其异构化带来的分子形状上的改变, 芳香族偶氮类化合 物广泛的应用于光控开关和超分子化学领域. 一般情况 下, 反式的偶氮苯分子由于构象比较稳定, 能量较低, 是分子的热稳定态, 将芳香族偶氮化合物接枝在单层膜

*E-mail:mqzhu@hust.edu.cn

Received October 8, 2012; revised December 5, 2012; published online December 20, 2012.

Project supported by the National Basic Research Program of China (No. 2013CB922104), the National Natural Science Foundation of China (Nos. 20874025, 21174045), the National Natural Science Foundation of China Research Fellowship for International Young Scientists (No. 21150110141), the China Postdoctoral Science Foundation (No. 20100480065), the Open Program for Beijing National Laboratory for Molecular Sciences (BNLMS) and the Special Financial Grant from the China Postdoctoral Science Foundation (No. 2012T50642).

国家基础研究计划(No. 2013CB922104)、国家自然科学基金(Nos. 20874025, 21174045)、国家自然科学基金委外国青年学者研究基金(No. 21150110141)、中国博士后科学基金(No. 20100480065)和中国博士后特别资助基金(No. 2012T50642)资助项目. 


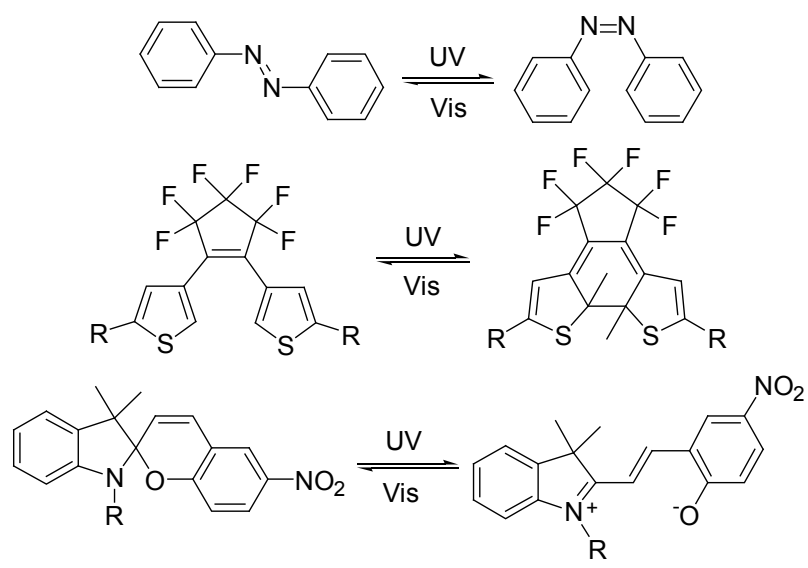

Scheme 1

中可以通过紫外光照射来改变膜表面的性质 ${ }^{[10]}$

二芳基乙烯和俘精酸酕类光致变色材料是通过周 环反应而产生开环态和闭环态. 通常其光异构化反应过 程中, 不产生活泼的自由基、离子或者偶极体, 因此分 子的热稳定性和抗疲劳性质较好. 其中全氟环戊烯为母 体的二芳基乙烯类材料由于其良好的热稳定性和抗痑 劳性而备受国内外研究者的关注 ${ }^{[1,11]}$.

螺吡喃和螺噁嗪是目前研究最广泛的光致变色化 合物 ${ }^{[12]}$. 分子中的吲哚啉环和苯并吡喃环通过中心处 的螺碳原子相连接, 因而两个环相互正交, 不存在共轭. 在近紫外或紫外光 $(<410 \mathrm{~nm})$ 激发下, 分子中螺环处的 碳一氧键发生异裂, 继而分子的构象和电子的排布发生 了很大变化. 螺碳原子从 $\mathrm{sp}^{3}$ 结构逐步转变为 $\mathrm{sp}^{2}$ 结构, 两个环系由正交型变为平面型, 分子的紫外-可见吸收 光谱表现为 $500 \sim 600 \mathrm{~nm}$ 处出现一个强的吸收峰, 分子 自此变为有色的开环态-部花菁. 在光和热的作用下, 部花菁分子又可以可逆的转变回无色的闭环态螺吡喃.

近年来, 超分辨显微成像技术成为一个研究热点, 这项技术突破了传统光学显微镜 $200 \mathrm{~nm}$ 的光学分辨率, 使得人们可以在更精细的分子水平上研究细胞生命活 动的相关过程. 在基于单分子定位的超分辨显微技术 中，通过一定的控制手段使得荧光探针可以在不同的荧 光状态之间转换: 在一定时间内只有稀疏的苂光分子处 于亮态，通过对这些亮着的分子进行单分子精确定位, 往复循环之后将这些精确定位出来的分子信息进行叠 加, 从而得到超分辨图像. 因此在这个过程中光学探针 扮演着至关重要的角色, 在一定程度上也决定了超分辨 成像的成像分辨率. 成像的分辨率不仅与实验条件和算 法有关，而且新的荧光探针的出现也会促进成像分辨率 的提高而促进生物医学的研究. 为了满足不断发展的新 型苂光显微镜的需求, 合成和发展与之相匹配的具有光 敏性的或具有光开关荧光团显得尤为重要. 而现有荧光 探针主要分为三大类: 荧光蛋白、光敏有机分子和光开
关纳米颗粒. 苂光蛋白分为三类: 光激活荧光蛋白、光 转换荧光蛋白和光开关荧光蛋白. 光激活苂光蛋白在光 照条件下从无色状态激发为明亮状态 ${ }^{[13]}$, 而光转换苂 光蛋白根据光刺激改变荧光发射波长 ${ }^{[14]}$, 光开关荧光 蛋白则是在特定激发下可以可逆控制开关状态 ${ }^{[15]}$. 理 想的荧光蛋白需要在光调节苂光的开和关或者调节两 种不同颜色方面拥有良好的可逆性，同时也需要容易的 进行光活化/光转换/光开光以得到高的成像对比度. 将 人工合成的光敏性有机小分子苂光团特异性的标记在 蛋白上，我们同样可以得到光敏性荧光蛋白 ${ }^{[16]}$. 而可以 用于光响应成像的能够光开光激活-受体分子对十分有 限 ${ }^{[17]}$, 如 Cy3/Cy5s. 所以我们亟须研究、设计和开发一 些代表性的具有可逆荧光光开关性质的光敏性有机分 子或纳米结构用于化学生物学相关领域的研究. 近年来 新发展的含螺吡喃的光敏纳米颗粒作为一种新型的苂 光探针, 以其优越的光化学和光物理性质, 正在引起大 家的关注. 本文主要聚焦于螺吡喃分子光开关材料的合 成和相关的特殊性质的研究及其在相关领域中的应用.

\section{1 螺吡喃的变色性质}

螺吡喃和螺噁嗪分子结构类似, 是一类研究最早, 最广泛的有机光致变色分子. 虽然早在 1921 年就已发 现了螺吡喃分子的热致变色性质，但是直到 1952 年 Fisher 和 Hirsherg 才发现它的光致变色性质. 近几年通 过设计合成特殊结构的螺吡喃分子，以此分子为骨架， 研究发现其除了具有典型的光致变色性质之外，还具有 其他的一些特殊性质. 例如: 力致变色性质, 电致变色.

\section{1 螺吡喃的力致变色性能}

Davis 等 ${ }^{[18]}$ 合成了螺吡喃衍生物 $\mathbf{1} \sim \mathbf{6}$ (Scheme 2), 研究了在外力作用下螺吡喃分子的变色行为(Eq. 1). 将 溴异丁酰溴与化合物 $\mathbf{1}$ 通过酯化反应得到化合物 $\mathbf{2}$, 即 在螺吡喃分子 $\mathbf{2}$ 的吲哚环上和吡喃环上分别引入了溴异 丁酸脂，化合物 2 通过原子转移自由基聚合(ATRP)的方 法引发丙烯酸酯聚合, 在得到的聚合物中螺吡喃分子作 为桥梁连接着两条聚合物链段, 而这两条聚合物链段也 正好位于螺环的两端. 将聚合物加工成亚铃型状之后, 对聚合物进行拉伸测试: 发现在机械外力的作用下，聚 合物链开始受到应力而被拉伸, 聚合物链进一步被拉伸 之后, 处于聚合物链中间的螺吡喃分子同样也受到了外 力作用, 分子中螺环处的 $\mathrm{C}-\mathrm{O}$ 键发生断裂，此时在分 子水平上, 无色态的闭环体螺吡喃转变为有色态的开环 体部花菁, 其宏观表现是整个聚合物由淡黄色转变为紫 红色. 同样发生的变色现象说明此类螺吡喃化合物是一 种具有机械响应性的智能有机分子. 


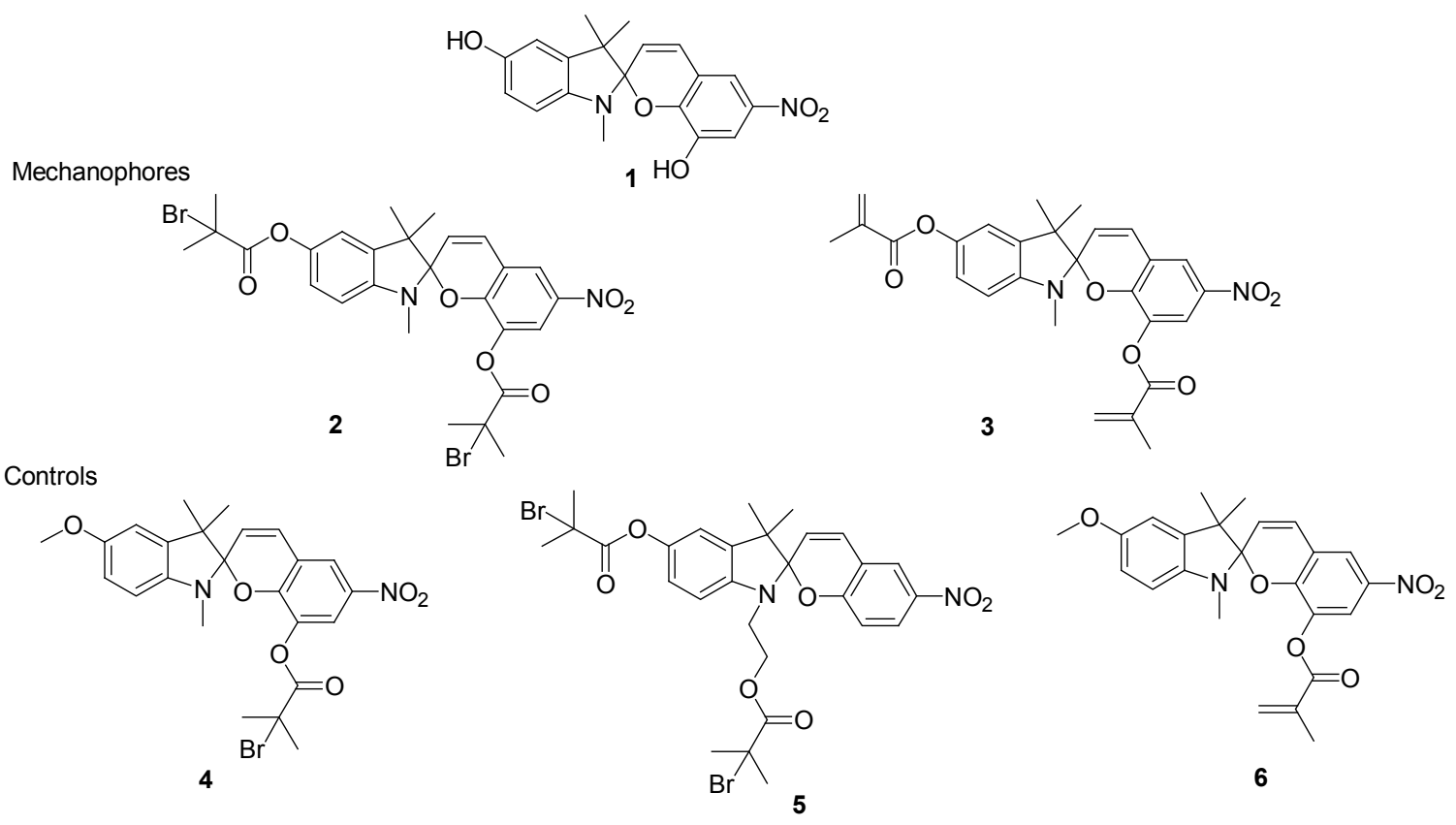

Scheme 2

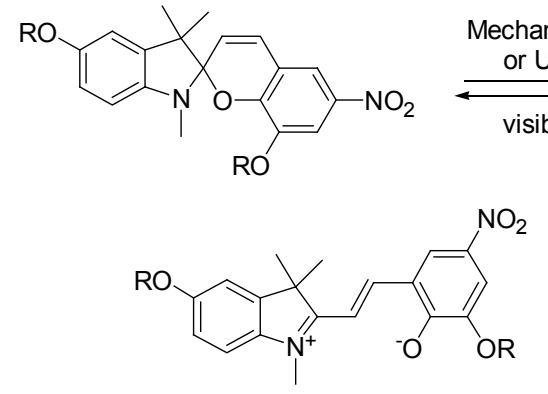

通过类似的方法得到的化合物 $\mathbf{3}$ 是在螺吡喃分子相
同的位置引入了甲基丙烯酸酯，通过本体聚合的方法得 到类似的聚合物. 同样的, 两条聚合物链段也位于螺环 的两端. 而对照组的化合物 4 , 螺吡喃分子上仅含有一 个溴异丁酸脂; 化合物 $\mathbf{5}$ 中两个溴异丁酸脂位于螺环的 同侧; 化合物 6 中螺吡喃分子上仅含有一个甲基丙烯酸 脂，因此在聚合之后得到的聚合物的链段没有分布在螺 吡喃分子螺环的两侧, 导致在相应的应力作用下, $4 \sim 6$ 对应的高分子没有观察到相应的力致变色现象.

O'Bryan 等 ${ }^{[19]}$ 合成了含有双羟基的螺吡喃分子 7 (Scheme 3). 分子 7 中羟基的位置分别位于螺吡喃分子
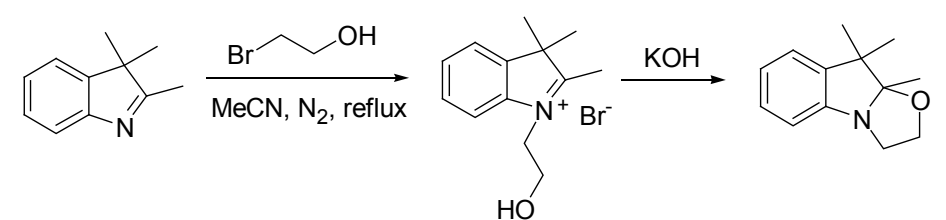

EtOH, reflux<smiles>Cc1cc([N+](=O)[O-])ccc1O</smiles>

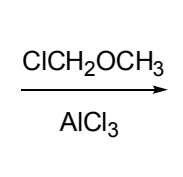<smiles>O=Cc1cc([N+](=O)[O-])cc(CCl)c1O</smiles><smiles>O=[N+]([O-])c1cc(Cl)c(O)c(CO)c1</smiles><smiles>CC1(C)c2ccccc2N(CCO)C12C=Cc1cc([N+](=O)[O-])cc(CO)c1O2</smiles><smiles>C1CCCCC1</smiles><smiles>[R]OCc1cc([N+](=O)[O-])cc2c1OC1(C=C2)N(CCO)c2ccccc2C1(C)C</smiles>

$\mathrm{R}=$<smiles>CC(=O)CCCCCOC(=O)CCCCCO</smiles>

Scheme 3 
螺环的两侧, 以辛酸亚锡为助催化剂, 引发了己内酯的 开环聚合. 同样的, 将包含螺吡喃的聚己内酯膜拉伸, 由于外力的传递, 位于螺环上 $\mathrm{C}-\mathrm{O}$ 键同样在受到外力 作用下而发生断裂，在宏观上表现为己内酯膜在要断裂 前, 颜色也随之发生了明显的变化, 螺吡喃分子表现出 力致变色的性质. 他们进一步利用密度泛函理论和时间 依赖的密度泛函理论也证明是由于螺环上较弱的 $\mathrm{C}-\mathrm{O}$ 键断裂而产生了有色态的部花菁, 进而表现为聚合物膜 的颜色也随之发生了变化.

将螺吡喃分子螺环两侧通过共价键连的方式嵌入 在聚己内酯、丙烯酸甲酯等聚合物链中, 得到了一类新 型的具有机械响应性的功能高分子, 而且理论和实验证 实是由于机械力诱导产生的部花菁导致的机械变色, 但 是由于聚甲基丙烯酸甲酯的机械强度高、柔韧性差、玻 璃化转变温度高 $\left(105{ }^{\circ} \mathrm{C}\right)$; 聚丙烯酸甲酯的机械强度差, 柔韧性高, 但其玻璃化转变温度较低, 约为 $6{ }^{\circ} \mathrm{C}$. 这些 聚合物不适合在室温下研究本体聚合物中有机械力诱 导产生的螺吡喃的动力学和热力学转变行为. Lee 等 ${ }^{[20]}$ 合成通过逐步聚合法得到了同样具有力学响应性的聚 氨酯-螺吡喃高分子, 依赖于聚氨酯的玻璃化转变温度 低，在实验中，室温下易到达平衡时间的特性，借助于 可见光光谱仪他们研究了机械力激活下螺吡喃转变为 部花菁的动力学和部花菁转变为螺吡喃的热力学性质. 进一步的研究表明随着拉力的增大, 可见光区部花菁的 吸收值也在增加. 而聚合物在一定的张力作用下, 转变 后的部花菁分子将不能转变回到原来的热力学稳定的 螺吡喃形式, 这也说明在这种机械响应性的聚氨酯中, 一定的张力可以改变聚合物中螺吡喃结构单元的势能 面.

\section{2 螺吡喃的电致变色性能}

聚噻吩作为一种常见的导电高分子, 以其独特的电 学, 光学性质引起了人们广泛的研究并逐步将其应用于 有机场效应晶体管 ${ }^{[21]}$ 、太阳能电池 ${ }^{[22]}$ 、化学传感 ${ }^{[23]}$ 以及 电致变色器件 ${ }^{[2]]}$ 等领域. 将螺吡喃光致变色的特性与噻 吩衍生物本身的电学、光学性质结合在一起, Wagner 等 ${ }^{[25]}$ 首次合成了化合物 9 (Scheme 4), 并通过电聚合得 到 poly-TThSP1. 在一定范围内, 该聚合物在不同电位 下能够呈现不同的颜色, 且电化学性质非常复杂. 为了 理解其反应机制, 他们仔细研究了 $\mathbf{8}$ 的电化学性质, 发 现 8 首先发生不可逆的电氧化开环反应得到至少二个氧 化态的部花菁同分异构体，部花菁同分异构体又可以继 续进行可逆的氧化还原反应. 该种性质也在 polyTThSP1 聚合物上有体现，当电压小于 $0.6 \mathrm{~V}$, 该聚合物 上的螺吡喃不会发生反应，发生的是聚噻吩的氧化还原 反应. 然而当电压大于 $0.8 \mathrm{~V}$, 该聚合物上的螺吡喃会发
生氧化, 不可逆的异构化得到氧化态的部花菁. 进一步 光电化学研究发现聚合物的电化学和物理性质主要与 部花菁及其是否有能力形成 $\pi$ 二聚体，低聚物或 $\pi$ 极性 阳离子二聚体有关(Scheme 5).
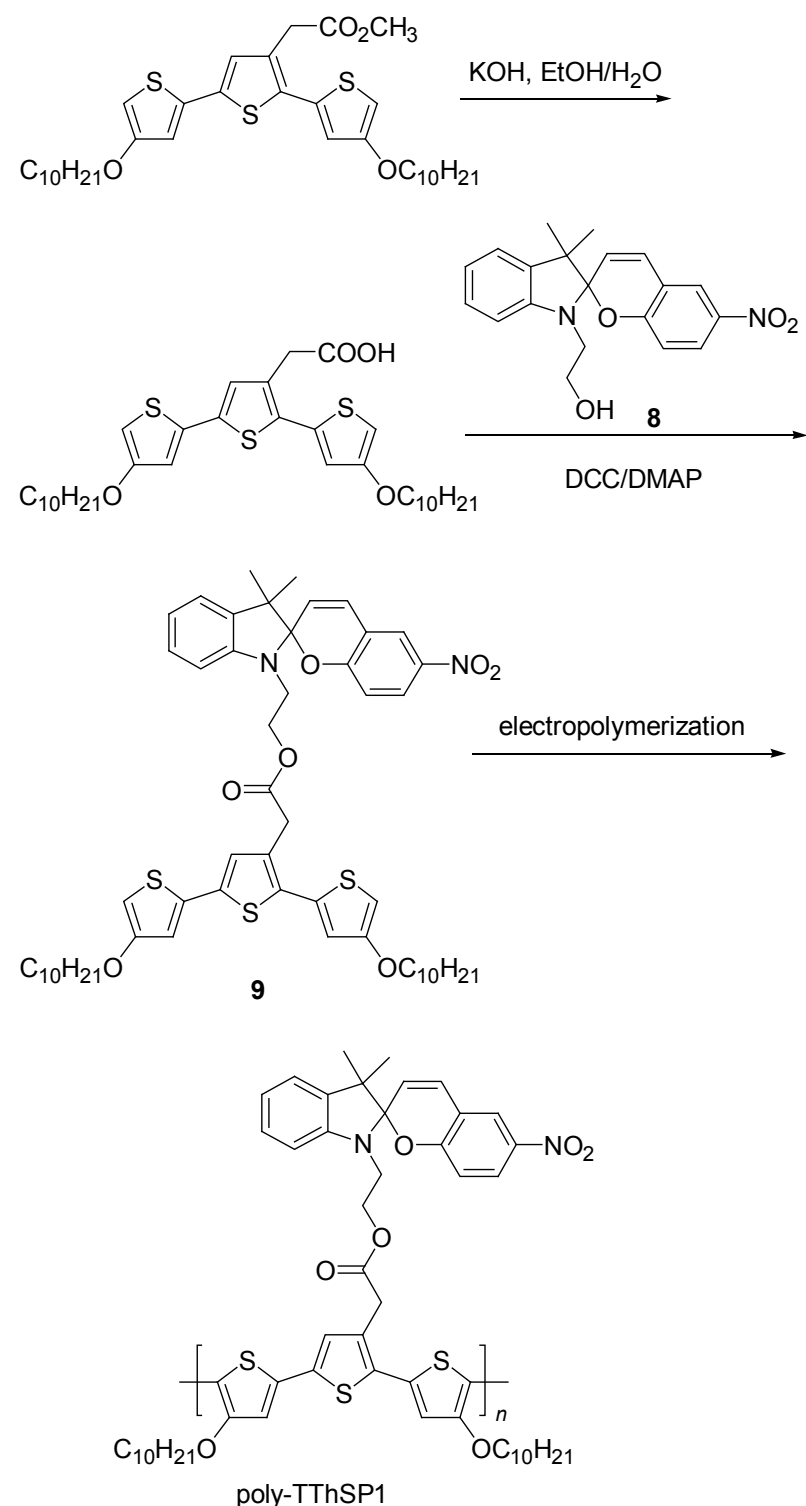

\section{Scheme 4}

他们的研究指出部花菁分子被氧化之后, polyTThSP1 薄膜的光化学行为就显著的受电化学性质的影 响. Poly-TThSP1 的氧化还原可以用来控制聚合物的光 化学行为: 即电压改变前后聚合物的导电性和先前的聚 噻吩相比变化不大，但是螺吡喃和部花菁的构象上的大 变化将会引起聚合物薄膜的形态的显著变化. 利用螺吡 喃和部花菁的物理化学性质变化引起聚合物体系为疏 水性，离子和水络合作用以及构象效应的变化为下一步 多功能材料的发展提供了一个很好的平台. 


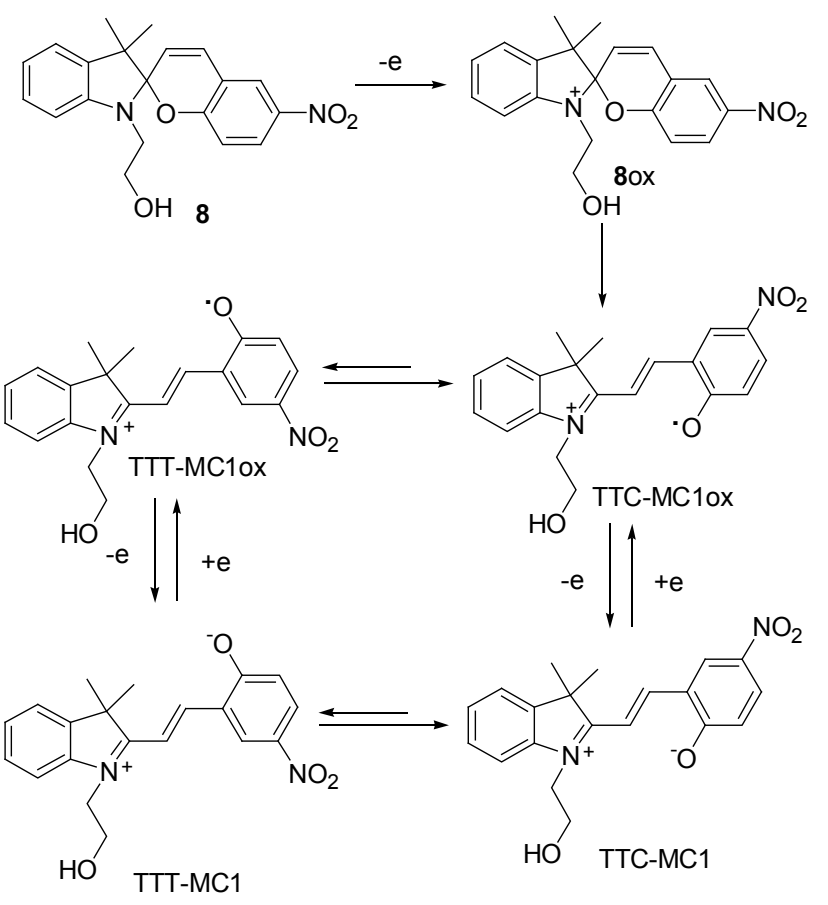

Scheme 5

\section{2 含光敏基团的螺吡喃分子}

Bahr 等 ${ }^{[26]}$ 合成螺吡喃衍生物 $\mathbf{1 0} \sim \mathbf{1 2}$ (Eq. 2), 其中 11 是含锌离子的卟啉-螺吡喃和 12 是不含锌离子的卟啉 一螺吡喃光致变色化合物. 他们研究了紫外光照前后光 开关分子对卟啉化合物的单线态能量的影响. 在这些染 料中, 螺吡喃是光激活的 “开关”, 并控制着卟啉第一 激发单线态的寿命. 当用可见光激发卟啉, 闭环体螺吡 喃不影响卟啉的激发态. 而用近紫外光激发螺吡喃或者 卟啉染料导致产生了部花菁, 伴随这 $600 \mathrm{~nm}$ 左右有一 吸收峰. 紫外光照前后卟啉单线态的寿命分别为 $1.8 \mathrm{~ns}$
和 $120 \mathrm{ps}$, 卟啉寿命的降低可能是卟啉单线激发态到部 花菁单线激发态的能量转移导致卟啉的猝灭.

二苯甲酮和慕类化合物及其衍生物是常用的光敏 剂, 基于分子间三线态到三线态的能量转移机制, 它们 可以敏化硝基螺吡喃的敏化光化异构反应. 基于此, Tomasulo 等 ${ }^{[27]}$ 通过将荎或二苯甲酮及其衍生物通过共 价键连的方式合成了螺吡喃衍生物 $\mathbf{1 3 \sim 1 8}$ (Scheme 6), 通过研究发现在同样实验条件下，化合物 $15 \sim 18$ 上的 光敏化结构单元并没有预期的促进敏化过程，比相应的 硝基螺吡喃 13,14 中的光异构化反应效率低 2 到 100 倍. 此外化合物 $15 \sim 18$ 光异构反应伴随着不可逆的转变, 敏化基团的菼光也被光致变色体系抑制. 进一步的实验 结果表明光致变色单元有效地抑制了敏化单元三线态 激发分子的数量和分子内三线态一三线态的能量转移, 而且分子内的相互作用促进了发光团-发色团这一系统 的光降解过程，即电子从发光团-发色团组合中敏化单 元到光致变色单元的分子内转移可能导致了光异构化 过程的不可逆. 综上所述, 他们研究表明将菜或二苯甲 酮敏化剂和硝基螺吡喃分子连接在同一共价骨架上来 提高这一体系的光生色效率不是一个很好的策略.

BODIPY 是最近二十年发展起来的一类荧光染料, 它以其独特的光物理和光化学性质, 例如高的苂光量子 产量和摩尔消光系数, 光谱半峰宽较窄, 对样品 $\mathrm{pH}$ 以 及溶剂不敏感、光稳定性好等受到广大科研工作者的亲 睐，并在离子和小分子探针，生物分析方面有重要应 用 ${ }^{[28]}$. Tomasulo ${ }^{[29]}$ 等将 BODIPY 单元与螺吡喃分子通过 烷基链连接得到了化合物 19 (Eq. 3), 研究了紫外光照 前后 BODIPY 单元与光致变色单元之间的能量转移情 况和螺吡喃分子的抗疲劳性质.

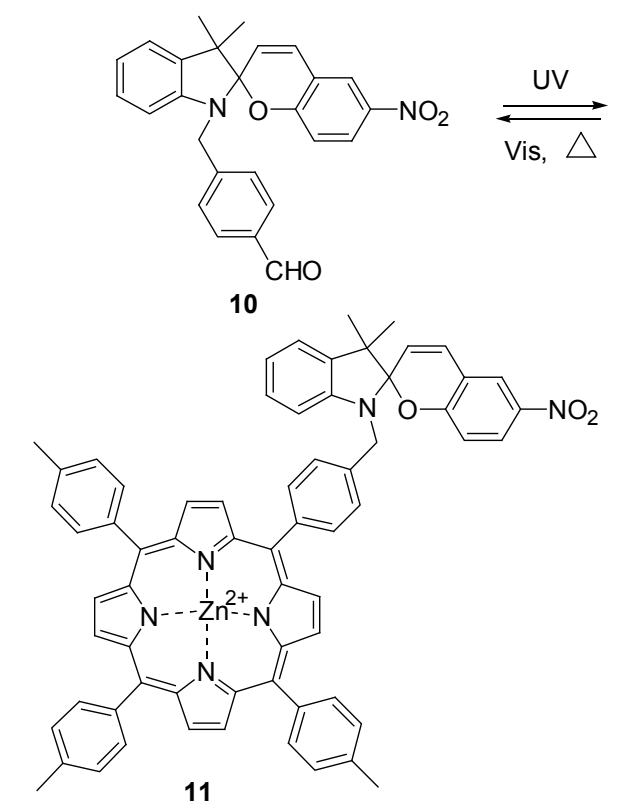




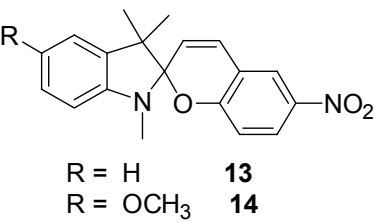

$\mathrm{R}=\mathrm{OCH}_{3} \quad 14$

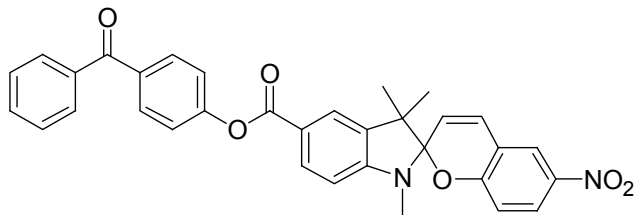

17

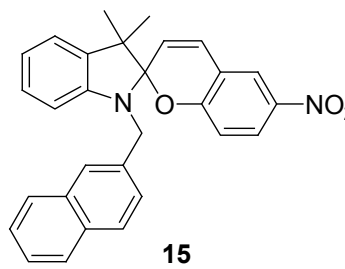

15
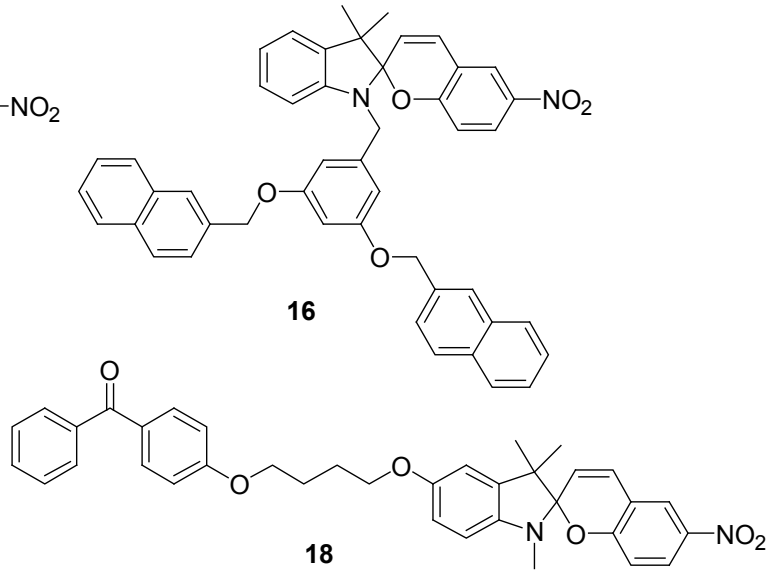

Scheme 6

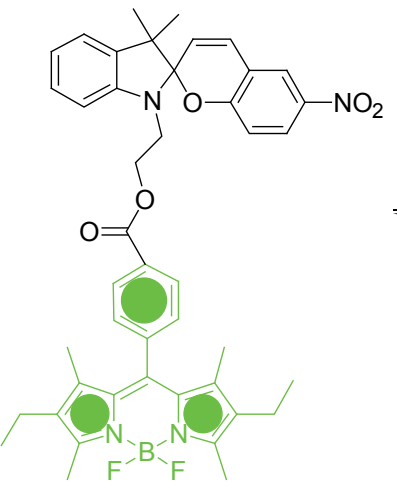

19
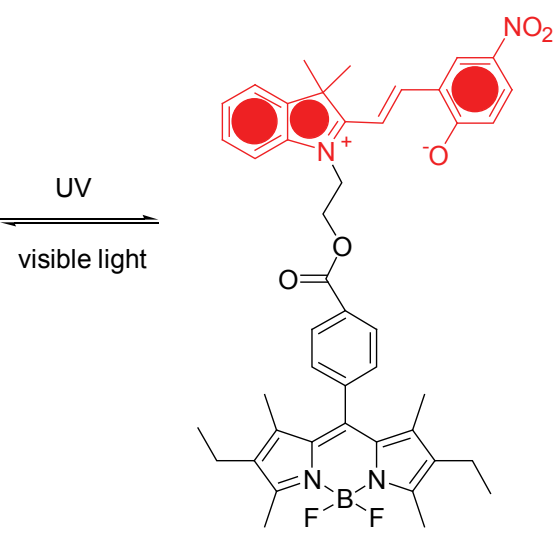

(3)

相连，阐释了光开关分子可以用于半导体纳米晶体苂光 的调控. 但是紫外光的照射引起了纳米晶体表面的光开<smiles>CC1(C)c2ccccc2N(CCCCCS)C12C=Cc1cc([N+](=O)[O-])ccc1O2</smiles><smiles>CC1(C)c2ccccc2N(CCCCCS)C12C=Cc1cc([N+](=O)[O-])ccc1O2</smiles><smiles>CCN1c2ccccc2C(C)(C)C12C=Cc1cc([N+](=O)[O-])ccc1O2</smiles>

20

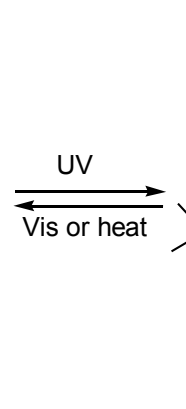<smiles>O=[N+]([O-])c1ccc(Oc2ccc([N+](=O)[O-])cc2)cc1</smiles><smiles>O=[N+]([O-])c1cccc(Cl)c1</smiles>

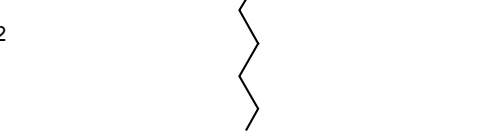<smiles>CCN1c2ccccc2C(C)(C)C12C=Cc1cc([N+](=O)[O-])ccc1O2</smiles><smiles>CCN1c2ccccc2C2(CC)CCCC12[O-]</smiles><smiles></smiles> 
关分子发生光降解, 导致其调控效果下降. 原因可能是 在三线态的氧分子、自由基或者紫外光照的影响下, 部 花菁分子均可能发生副反应而导致分解，因此合成一些 新的具有良好抗疲劳性质的螺吡喃光开关分子, 或将螺 吡喃分子置于厌氧环境, 如疏水性的空腔中, 抑或在纳 米晶体表面添加一些可以猝灭三线态氧分子或者自由 基的染料分子 ${ }^{[31]}$ 将有助于改善其调控性能.

\section{3 螺吡喃对离子或分子的识别}

在从闭环态的螺吡喃形式转变为开环的电荷分离 的双离子形式的部花菁结构, 分子的偶极距显著增加. 因此可以利用螺吡喃的这一特殊性质控制溶液中或修 饰之后的颗粒表面上螺吡喃单元和带电荷体之间的静 电相互作用, 达到离子识别的目的. 冠醚以其特殊的空 腔大小可以与一些金属离子进行结合, 例如 15-冠-5 与 钠离子的识别, 18 -冠- 6 与钾离子的识别等. 等将冠醚连 接在螺吡喃分子上, 通过结合前后, 冠醚的构象变化使 得化合物结构发生了重组, 离子与冠醚结合后达到了最 稳定的构象. 完成了识别过程的构象重组. Inouye 等 ${ }^{[6]}$ 合成了含大小不同的单氮杂环苯并螺吡喃化合物 21a 21d (Eq. 5), 研究发现向化合物的已经溶液中加入碱金 属离子, 化合物 $21 \mathrm{~b}$ 对锂离子的识别能力最好.
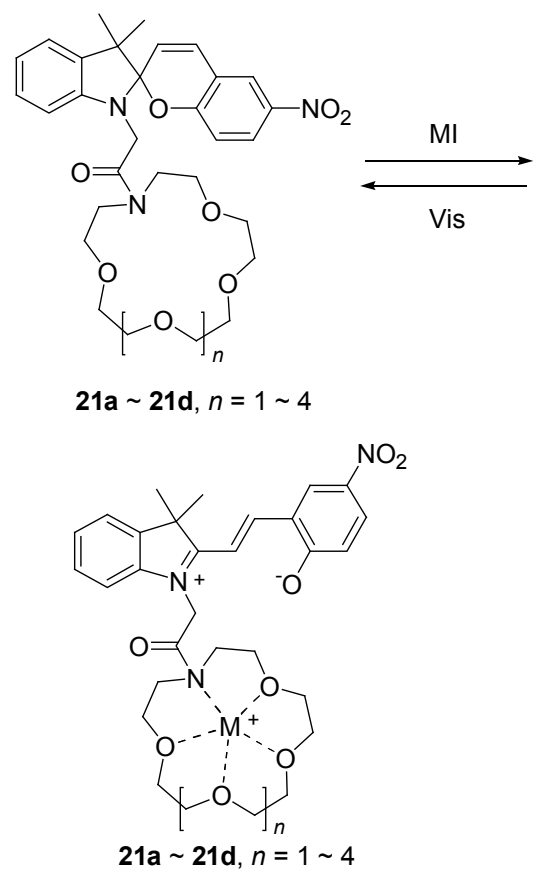

随后 Inouye 等 ${ }^{[32]}$ 进一步合成了化合物 22. 研究发 现只有在碱金属离子和紫外光的共同作用下, 才能够形 成部花菁一碱金属络合物(Eq. 6). 进一步的研究表明此 时的键金属离子是与分子中的氧乙炔链上的醚氧原子 相结合, 而且此络合物十分稳定, 在黑暗条件下放置一
个月也不会发生热异构化反应生成螺吡喃，即碱金属的 加入稳定生成的部花菁结构. 同时他们还预言了螺吡喃 的这种特殊性质使其可作为光控逻辑开关.
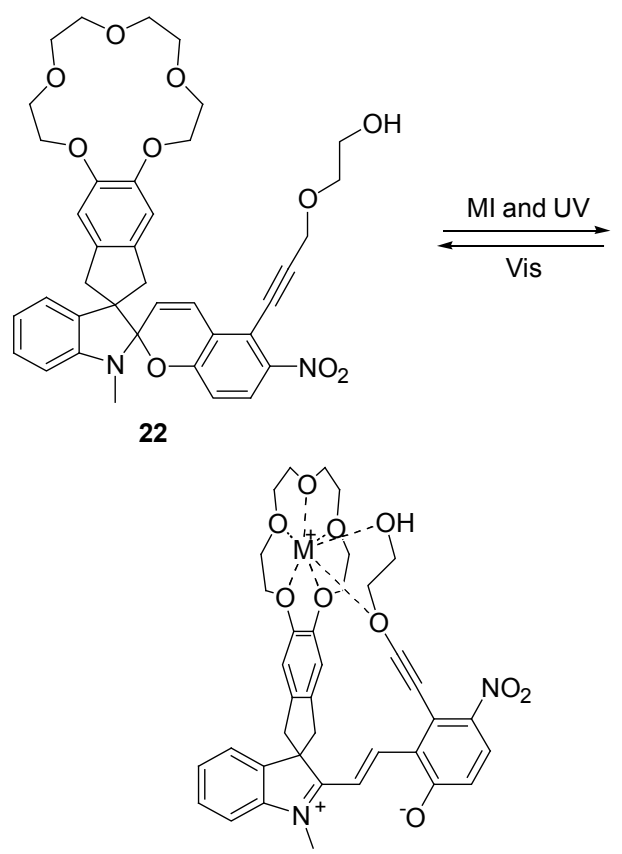

双(2-吡啶甲基)氨基是一个很好的检测锌离子的配 体 ${ }^{[33]}$. Shao 等 ${ }^{[34]}$ 设计合成含双(2-吡啶甲基)氨基的螺吡 喃分子 23 (Eq. 7), 在乙醇和水的混合体系中, 伴随着 $\mathbf{2 3}$ 与 $\mathrm{Zn}^{2+}$ 络合, 其在 $560 \mathrm{~nm}$ 处的苂光发射强度变低而在 $620 \mathrm{~nm}$ 处产生新的荧光峰. 加入阴离子焦磷酸根之后, 焦磷酸根与螺吡喃和 $\mathrm{Zn}^{2+}$ 进一步作用, 导致 $560 \mathrm{~nm}$ 处 的苂光强度增强，而 $620 \mathrm{~nm}$ 处荧光强度降低. 有趣的是 随着焦磷酸根浓度的增加，两波长处苂光强度之比与焦 磷酸根的浓度成正比，以此他们实现苂光比对法对焦磷 酸根的定量检测分析. 进一步的研究表明其形成的络合 物是基于金属协同配位作用, 螺吡喃、锌离子、焦磷酸 根形成了一个 $2: 2: 1$ 的三元络合物.
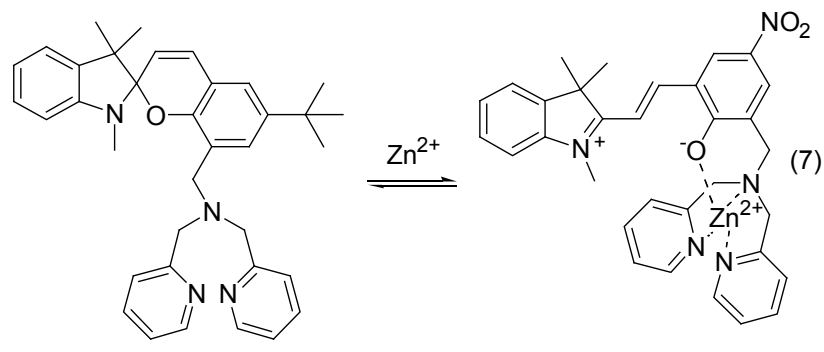

23

Shao 等 ${ }^{[35]}$ 合成了螺吡喃分子 $\mathbf{2 4 \sim 2 6}$ (Scheme 7), 分子 20 的开环态的部花菁结构与谷胱甘肽分子之间在 空间上具有结构互补的主-客体效应上的而且多静电位 点的相互作用, 使其可以作为新型的苂光探针用于活体 中谷胱甘肽的检测, 同时避免了其他含硫组分的干扰. 
不需要通过其他的转染技术, 化合物 20 就可以在细胞 内传输, 并可以在细胞中富集, 利用荧光共聚焦显微成 像技术, 实现了对活细胞中谷胱甘肽分子的检测成像.

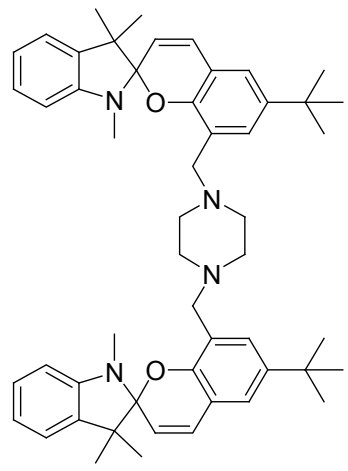

24

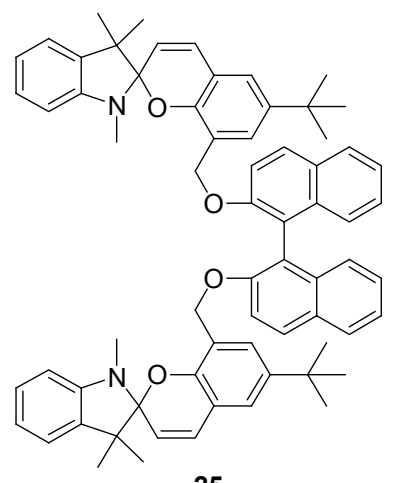

25

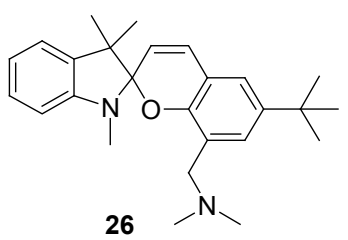

Scheme 7

Champagne 等 ${ }^{[36]}$ 合成了苯并噻唑-螺吡喃化合物 27 (Eq. 8), 其特有的二阶非线性性质使其具有有效的识别 阳离子大小和电荷的能力, 与碱金属、碱土金属、过渡 金属阳离子之后, 其结构和二阶非线性光学性质上产生 的差别, 而且自身的光致变色性质可以使其从复杂的形 式转变回到螺吡喃结构, 结合复合线性或者非线性光学<smiles>[R7]c1cccc2c1N(C)C(/C=C/c1cc(Cl)cc(-c3sc4ccccc4[n+]3C#N)c1OC)C2(C)C</smiles>

方法提高了阳离子检测的选择性，为环境监测提供了一 种可重复使用的探针. 通过利用螺吡喃到部花菁的光反 应异构可以用来调节配体探针的有效性，扩大了阳离子 监测的范围和有效性.

\section{4 光控逻辑开关}

荧光素由于其荧光量子产率高, 易于改性, 在多种 应用(如苂光抗体技术)中被广泛用作为苂光示踪物 ${ }^{[37]}$. Guo 等 ${ }^{[38]}$ 将荧光素与螺吡喃通过烷基链连接，设计了合 成含两个螺吡喃单元和一个荧光素单元的染料 28 分子 (Scheme 8). 由于部花菁的吸收光谱与苂光素的发射光 谱能够较好的匹配, 他们研究了用于研究单分子水平信 息传递. 基于共振能量转移机制, 新染料的荧光可以通 过三输入单元：紫外光(I1), 可见光(I2)和质子(I3)的调 整而改变(Scheme 9). 在三输入单元的协同作用下，分 子的发光行为与逻辑集成电路中集成了一个 “或” 和 “和” 逻辑开关相符合(表 1), 这为分子开关在复杂逻辑 开关中的应用奠定了一定的基础.

在过去的十几年中，菲酰亚胺类材料具有的特殊的 光化学和光物理性质, 使其衍生物在有机发光二极管 (OLED)、有机场效应晶体管(OFET)、太阳能电池、分 子开关、分子导线、荧光探针等领域一直成为广大科研 工作者研究的热点 ${ }^{[39]}$. Guo 等 ${ }^{[40]}$ 合成螺吡喃 14, 29 (Scheme 10). 化合物 29 螺吡喃和菲酰亚胺单元的光谱 和电化学性质在基态强烈地耦合导致了菲酰亚胺单元 的苂光几乎被猝灭. 他们发现染料 29 与紫外光(I1), 铁 离子(I2)，和质子(I3)协同作用下，利用 $480 \mathrm{~nm}$ 波长的可 见光激发, 芢酰亚胺单元又可以发射出荧光(Scheme 11). 而这种三因素的相互协同作用正好符合三输入单 元的逻辑门 “和” , 如表 2 所示. 他们的有趣发现为下 一步设计和构建复杂的由特殊光化学和光物理性质的 有机化合物构成的具有多功能的光电器件提供了新的 思路.
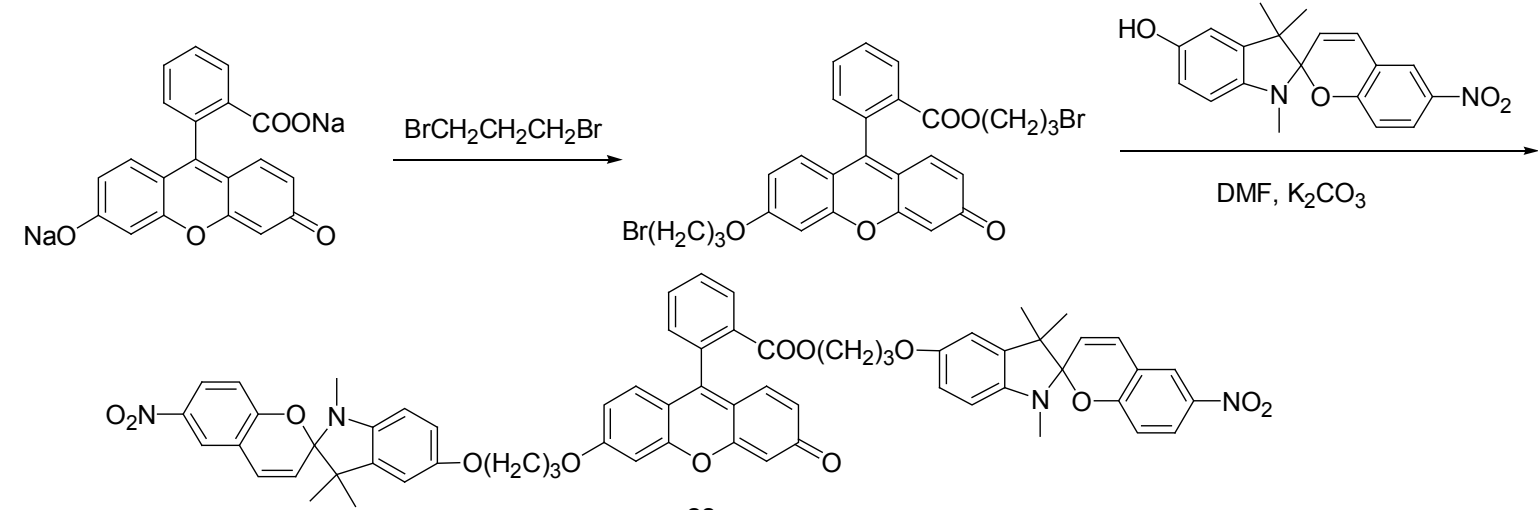

28

Scheme 8 


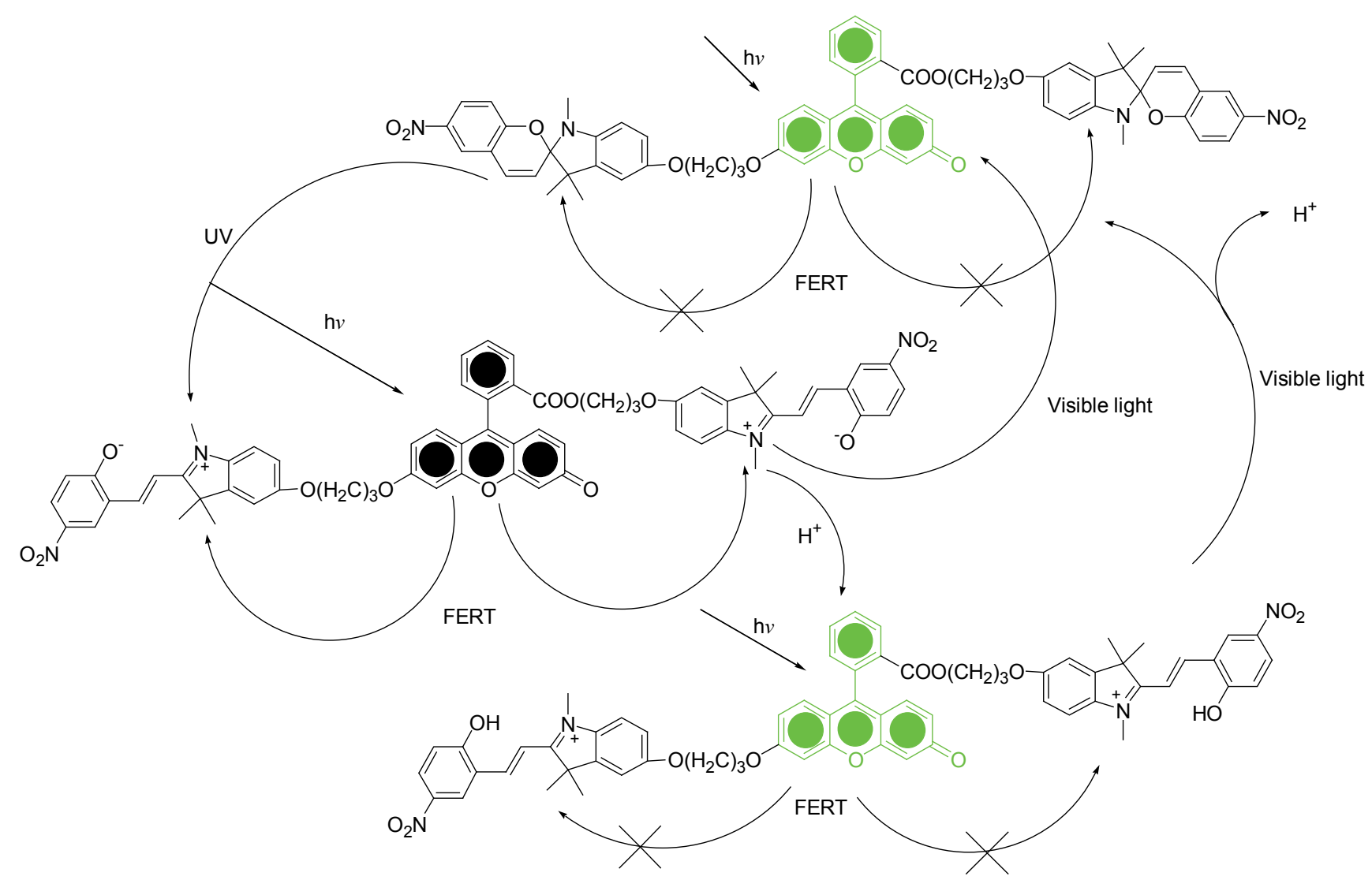

Scheme 9

表 1 化合物 28 在紫外光, 可见光和离子作用下逻辑输出

Table 1 Truth table of three binary inputs data and corresponding output digit

\begin{tabular}{|c|c|c|c|}
\hline \multicolumn{3}{|c|}{ Input } & \multirow{2}{*}{$\begin{array}{c}\text { Output } \\
\text { O1 }\end{array}$} \\
\hline UV ligft (I1) & Visible light (I2) & Proton (I3) & \\
\hline 0 & 0 & 0 & 1 \\
\hline 0 & 0 & 1 & 1 \\
\hline 0 & 1 & 0 & 1 \\
\hline 1 & 0 & 0 & 0 \\
\hline 0 & 1 & 1 & 1 \\
\hline 1 & 0 & 1 & 1 \\
\hline 1 & 1 & 0 & 0 \\
\hline 1 & 1 & 1 & 1 \\
\hline
\end{tabular}

表 2 化合物 29 在紫外光、可见光和离子作用下逻辑输出

Table 2 Truth table of three binary inputs data and corresponding output digit

\begin{tabular}{cccc}
\hline UV light (I1) & Iron I2 & Proton (I3) & O1 \\
\hline 0 & 0 & 0 & 0 \\
1 & 0 & 0 & 0 \\
0 & 1 & 0 & 0 \\
0 & 0 & 1 & 0 \\
1 & 1 & 0 & 0 \\
1 & 0 & 1 & 0 \\
0 & 1 & 1 & 0 \\
1 & 1 & 1 & 1 \\
\hline
\end{tabular}

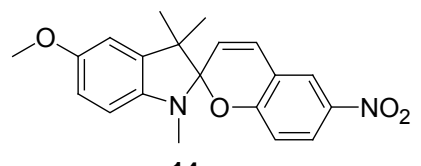

14

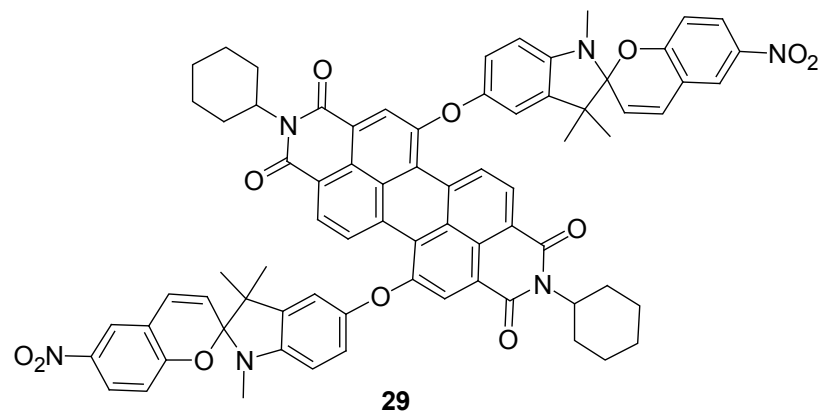



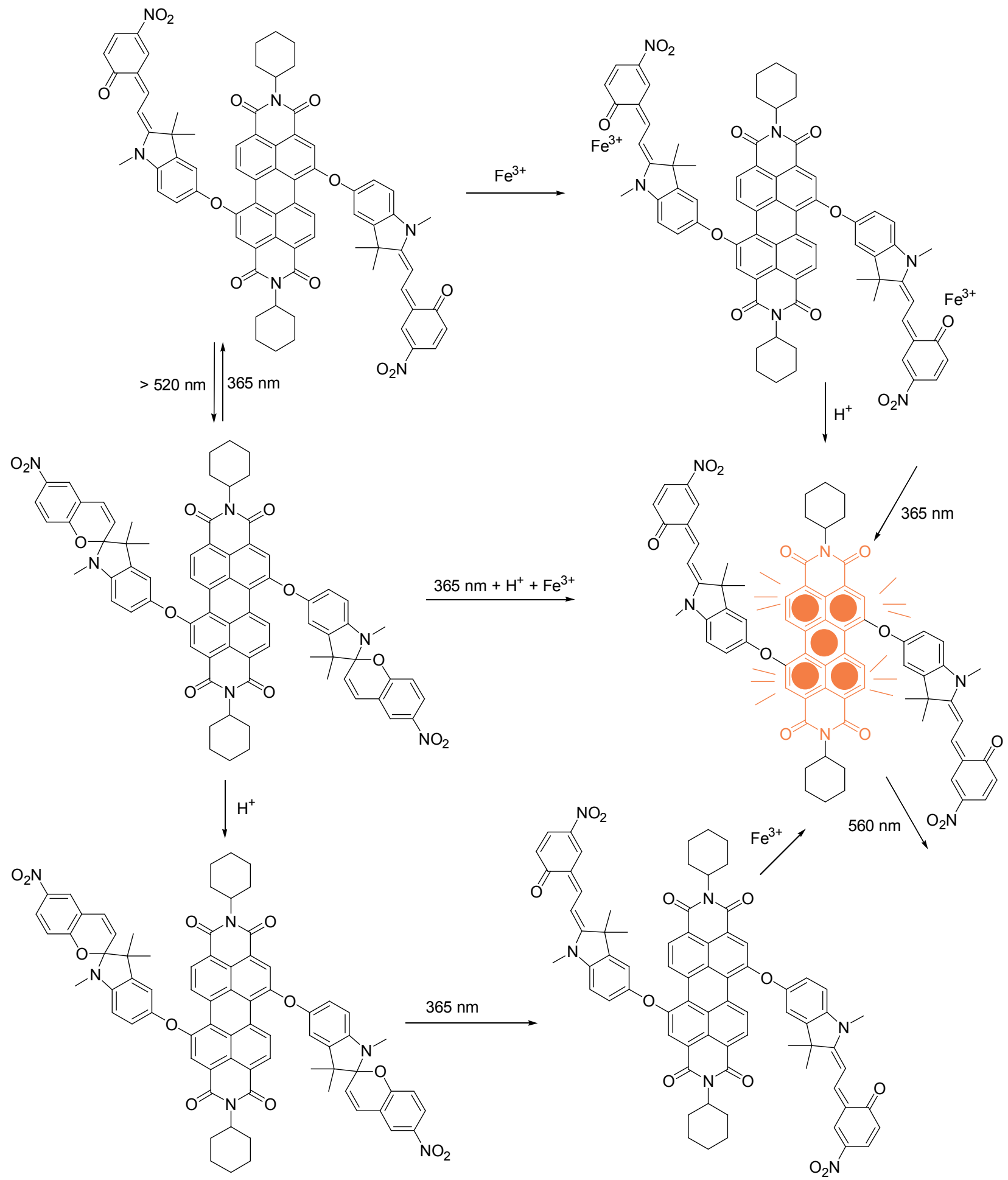

Scheme 19

\section{5 双光子过程及成像}

\section{1 双光子吸收和激发}

双光子激发的基本原理是：在高光子密度的情况 下，荧光分子可以同时吸收 2 个长波长的光子, 在经过 一个很短的激发态寿命的时间后, 发射出一个波长较短 的光子; 其效果和使用一个波长为长波长一半的光子去
激发荧光分子是相同的. 通常具有给体- $\pi$-给体，受体- $\pi$ 受体或者给体- $\pi$-受体结构的荧光团化合物有较大的双 光子吸收截面. Saita 等 ${ }^{[1]}$ 报道了合成了含两个二芳烯单 元具有给体- $\pi$-给体结构的化合物 31 (Eq. 9), 他们仔细 研究了此二芳烯衍生物的光致变色活性和双光子吸收 截面. 用 $820 \mathrm{~nm}$ 双光子经过短暂的照射之后，在被照射 


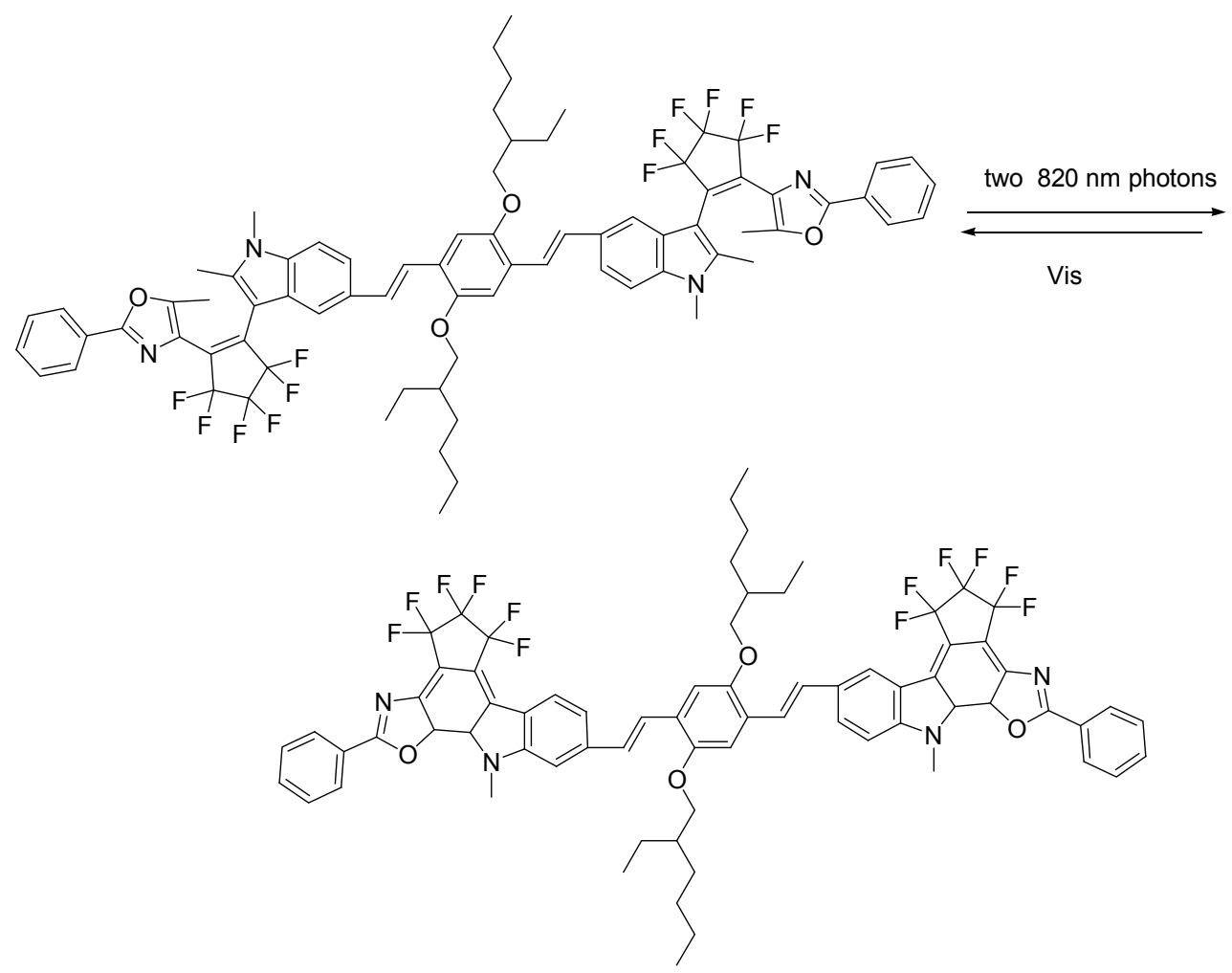

(9)

区域化合物由原来的开环态变为闭环态, 颜色也随之由 原来的黄色转变为绿色, 而尚未被照射的区域仍然保持 着黄色. 这说明化合物 31 能够吸收 $820 \mathrm{~nm}$ 的双光子而 进行从开环到闭环的结构转变. 相反用波长大于 600 $\mathrm{nm}$ 的可见光照射时, 此区域也会发生可逆的光致变色 反应，而绿色也会被擦除.

双光子激发成像使用的激光器是具有高能量锁模 的飞秒脉冲激光器 ${ }^{[42]}$. 这种激光器发出的激光具有很高 的峰值能量和很低的平均能量, 在使用高数值孔径的物 镜将脉冲激光的光子聚焦时, 物镜的焦点处的光子密度 是最高的, 双光子激发只发生在物镜的焦点上 ${ }^{[43]}$, 所以 双光子显微镜不需要共聚焦针孔, 提高了苂光检测效 率. Harada 等 ${ }^{[44]}$ 报道了俘精酸酤化合物 32 能够同时吸收 双光子以达到激发态(Eq. 10). 多数的俘精酸䣶化合物 在固态发生光致变色反应, 长波长的光穿透材料的深度 更深, 当用稳定的紫外光 $405 \mathrm{~nm}$ 照射晶体时, 入射光能 够开始穿过晶体的表面, 而晶体内部的结构未发生变 化. 与此情况相比, 当用 $742 \mathrm{~nm}$ 的激光脉冲照射晶体 时, 有色态的俘精酸䣶化合物在晶体中能够均匀的分 布, 说明在晶体中脉冲引起的光化学反应更深更均匀.

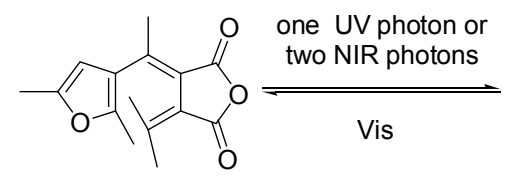

32

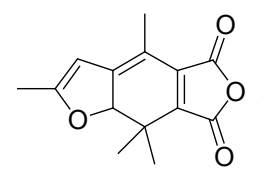
(10)
二茂铁及其衍生物是一类具有氧化还原中心的金 属有机化合物, 热稳定性好, 是很好的电子传递媒介体. Yuan 等 ${ }^{[45]}$ 合成了含二茂铁结构单元的螺噁嗪分子 33 (Eq. 11), 分子中二茂铁结构单元提高了光异构产生的 部花菁 34 的热稳定性, 二茂铁单元与部花菁醌式结构 34 可能存在的电相互作用以及其自身有效的三线态猝 灭能力导致了异构体 $\mathbf{3 4}$ 的稳定. 利用双光子技术, 他们 实现了高密度信息存储可重复擦写, 为下一步实现超高 密度的信息存储奠定一定的基础.

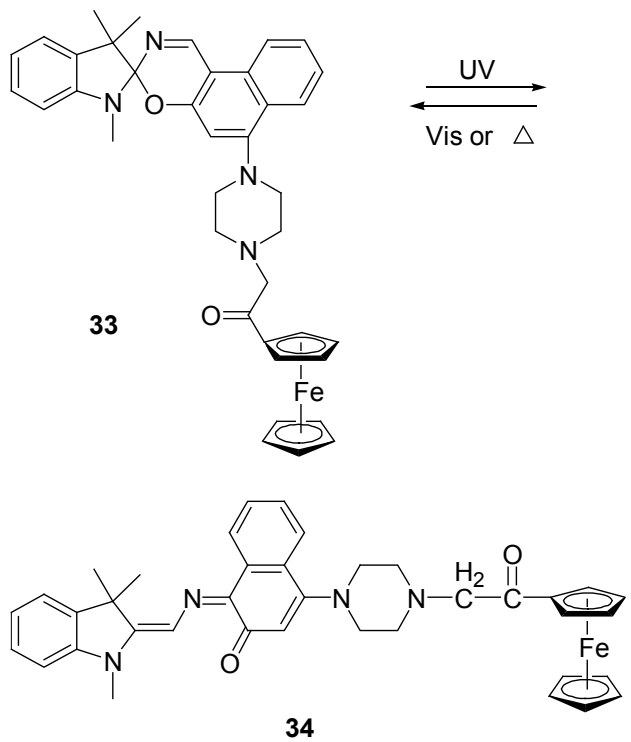




\section{2 双光子吸收与荧光成像}

Zhu 等 ${ }^{[46]}$ 合成了含两个丙烯酸酯的萠酰亚胺绿光单 体, 以此单体作为交联剂与化合物 35 (Scheme 12) 以及 其他乙烯基单体通过乳液聚合的方法得到具有红绿双 色可转换的光开关纳米粒子(图 1). 研究结果显示此纳 米颗粒的苂光强度高, 抗疲劳以及抗漂白性质好. 通过 紫外一可见光的交替照射可以实现红绿双色可逆转换, 实现了纳米颗粒的共定位作用, 消除了细胞中自发荧光 的干扰，为下一步追踪和标记复杂生物系统奠定了良好 的基础.

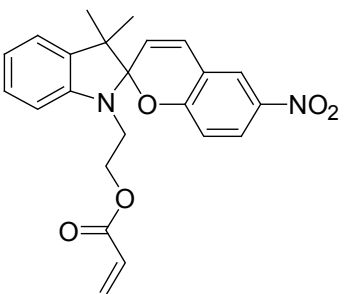

35

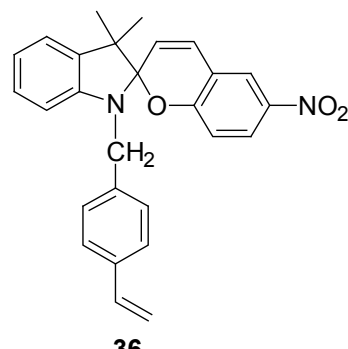

36
Scheme 12

Tian 等 ${ }^{[47]}$ 设计合成了含苯乙烯单元的系列螺吡喃 衍生物 36 39 (Eq. 12)(表 3), 光开关单体与苯乙烯、丙 烯酸正丁酯、丙烯酰胺等工具之后, 发现含 37 的纳米颗 粒在紫外光和可见光照射前后可以发射两种红绿荧光, 含 38 的纳米颗粒在紫外光和可见光照射前后可以发射 两种红和青色的荧光, 不需要借助其他荧光团的荧光, 单个纳米颗粒就以实现双色荧光的发射，提高了细胞内 成像的精度.

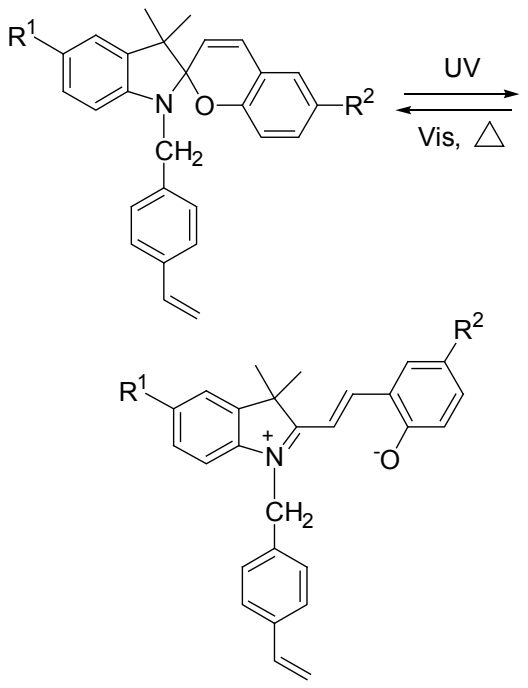

表 3 不同取代基对应的螺吡喃化合物

Table 3 Different substituents of spiropyran compounds

\begin{tabular}{ccc}
\hline Compd. & $\mathrm{R}^{1}$ & $\mathrm{R}^{2}$ \\
\hline $\mathbf{3 6}$ & $\mathrm{H}$ & $\mathrm{NO}_{2}$ \\
$\mathbf{3 7}$ & $\mathrm{MeO}$ & $\mathrm{NO}_{2}$ \\
$\mathbf{3 8}$ & $\mathrm{MeO}$ & $\mathrm{CN}$ \\
$\mathbf{3 9}$ & $\mathrm{H}$ & $\mathrm{CN}$ \\
\hline
\end{tabular}

由于受到衍射极限的限制，普通荧光显微成像的分 辨率都在 $300 \mathrm{~nm}$ 左右. $\mathrm{Hu}$ 等 ${ }^{[9]}$ 设计合成了含苯乙烯分 子的螺吡喃 36, 聚合后得到直径约为 $70 \mathrm{~nm}$ 左右的光开 关纳米颗粒，他们借助于光激活单分子逻辑开关获得结 构重建的显微技术(PULSAR)重建出了溶酶体中相邻的 两个光开关纳米颗粒的大小, 并通过高斯拟合得到了两 个颗粒间的间距。在尺度上生物的活动规律和机理 PULSAR 显微技术为生物成像提供了一种高分辨方法.

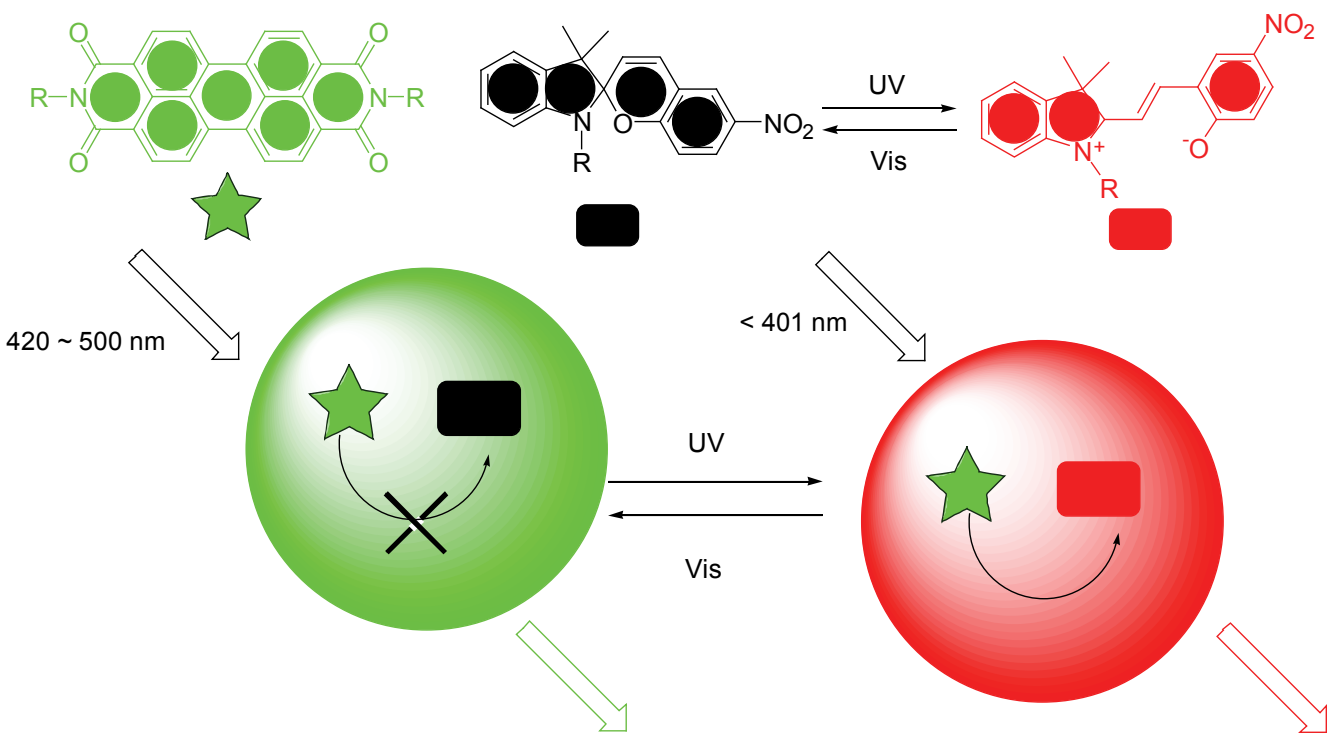

图 1 菲酰亚胺与螺吡喃分子之间的荧光共振能量转移(FRET)

Figure 1 The FRET between perylnene dyes and merocyanine dyes 
Tian 等 ${ }^{[48]}$ 同将光开关单体 36 与丙烯酸, 丙烯酰胺 苯乙烯等单体共聚, 借助于亲疏水性相互作用得到表面 含羧基的光开关纳米颗粒. 在 $\mathrm{CaCl}_{2}$ 表面自组装成单层 的光开关, 借助于螺吡喃分子的有效开关, 通过普通荧 光成像与光激活单分子逻辑开关获得结构重建显微技 术对比, 阐释了光激活单分子逻辑开关获得结构重建显 微技术可能应用于实时成像, 为活细胞中生物研究提供 了新的途径.

Zhu 等 ${ }^{[49]}$ 合成了含丙烯酸酯的螺吡喃分子 35 , 并将 其他乙烯基类小分子共聚, 得到直径为 $70 \mathrm{~nm}$ 左右的高 分子微球(Eq. 13). 因为聚合后螺吡喃分子位于高分子 微球的内部, 所以其抗疲劳性质, 循环开关性能和荧光 量子产率均有很大的提高. 他们将纳米微球通过脂质体 转染进入细胞后, 发现苂光性质和抗疲劳性质仍可以得 到保持, 为下一步生物标记和细胞成像奠定了良好的基 础.

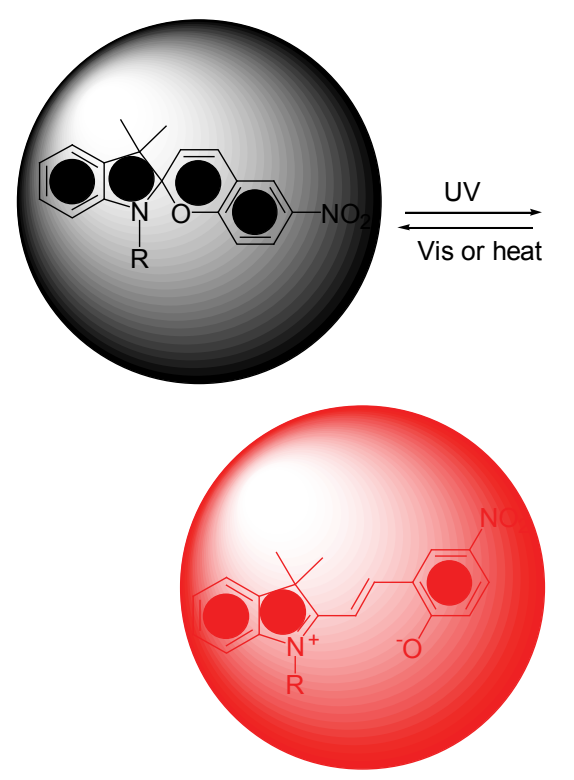

结合了激光扫描共聚焦显微镜和双光子激发技术 的双光子荧光显微镜采用的是可见光或近红外光(波长 为 $700 \sim 1200 \mathrm{~nm}$ ) 作为激发光源 ${ }^{[0]}$, 相对于紫外光、可 见光和近红外光都具有更强的穿透能力, 共聚焦苂光成 像的成像深度一般在 $50 \mu \mathrm{m}$ 左右, 而双光子显微镜的成 像深度可达 $1000 \mu \mathrm{m}$, 而且这一波段的光对活体细胞和 组织的光损伤小, 因而受生物组织散射的影响更小, 适 用于长时间的研究, 可以解决对生物组织中深层物质的 层析成像研究问题. 由于双光子吸收截面小, 只有在焦 平面很小的区域内可以激发出苂光, 故焦点以外的区域 都不会发生光漂白现象, 所以对材料的破坏性小. 与共 聚焦成像相比, 双光子成像不需要光学滤波器, 因而提 高了对荧光的收集率, 而收集率的提高也直接导致图像
对比度的提高. 因为被激发材料的荧光发射波长小于入 射波长, 与单光子激发相比, 许多有机小分子苂光探针 的多光子激发光谱变宽, 这样, 可以利用单一波长的激 发光同时激发多种染料, 从而得到同一生命现象中的不 同信息，便于相互对照、补充，并且大大降低了瑞利散 射的干扰产生的背景噪声. 生物组织中的自发荧光物质 的激发波长一般在 350 560 nm 范围内, 采用近红外或 红外波段的激光作为光源, 大大降低生物组织对激发光 的吸收.

基于双光子荧光显微技术的诸多优点, Zhu 等 ${ }^{[51]}$ 经 过进一步的研究, 将螺吡喃光开关纳米颗粒与抗-Her2 抗体连接, 之后与 SK-BR-3 细胞一起孵育成像. 在 780 $\mathrm{nm}$ 双光子照射下, 由于光开关纳米颗粒已经与目标的 抗-Her2 抗体免疫功能化, SK-BR-3 细胞膜上 Her2 膜受 体将会被红色苂光点亮. 相反, 在 $488 \mathrm{~nm}$ 的单光子激光 器照射下, 部花菁纳米颗粒将会回到螺吡喃纳米颗粒, 红光也会随之消失(图 2). 利用免疫功能化的颗粒和目 标 SK-BR-3 细胞作为模型, 可以有效地解释红色荧光能 够可逆地被双光子 “开” 并被单光子 “关” . 由于双光 子照射组织时, 对组织的破坏, 光毒性以及漂白等效应 可能都会减轻, 因此在生物成像应用方面光激活或光开 关的荧光探针, 利用近红外双光子可替代紫外光照射光 开关将会很有前景的.

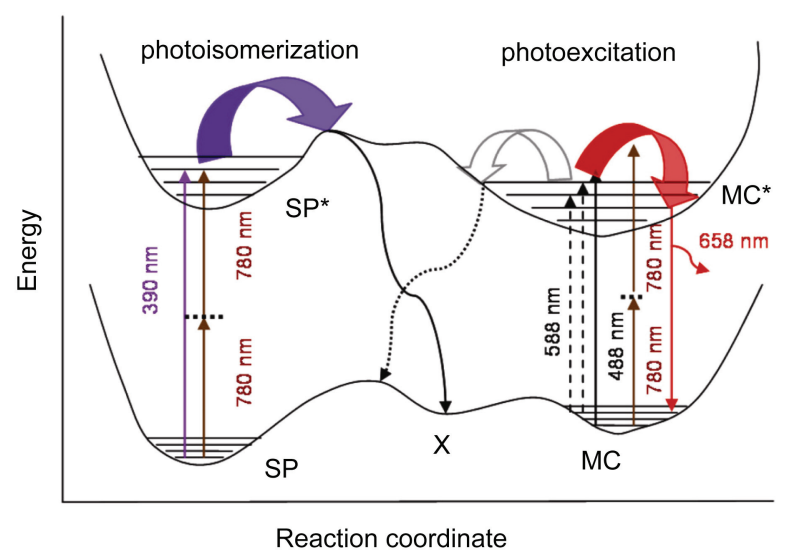

图 2 螺吡喃和部花菁之间光转化伴随的荧光过程

Figure 2 The fluorescence process caused by photoisomeration between spiropyran and merocyanine

Tan 等 ${ }^{[52]}$ 合成了等化合物(Scheme 13), 研究了不同 取代基对其光致变色性质的影响, 并研究了 SP-gPMMA 不同水解率对其热褪色性质的影响.

Pang 等 ${ }^{[53]}$ 合成系列烷基链含有 $\mathrm{N}$ 杂环的螺吡喃分 子(Scheme 14), 系统研究了这些分子在溶液中和聚合物 PMMA 和 PVB 薄膜中的变色和褪色过程以及耐疲劳性 质. 


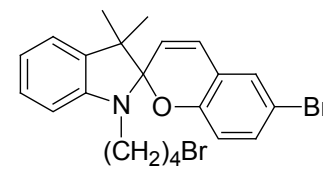

40
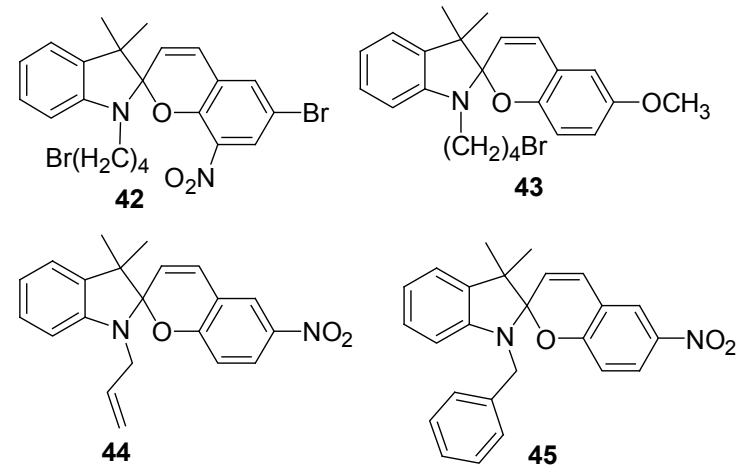

Scheme 13<smiles>[R]CCCN1c2ccccc2C(C)(C)C12C=Cc1cc([N+](=O)[O-])ccc1O2</smiles><smiles>[R]C1CCC([R6])CC1</smiles><smiles>CN1CCc2ccccc21</smiles>

49

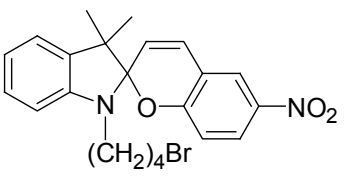

41

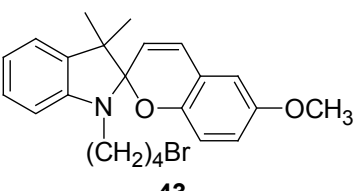

43

45

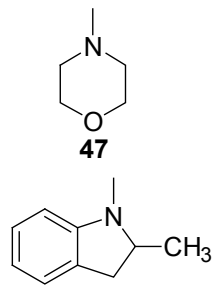

50

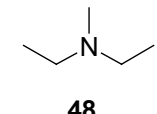

48<smiles>CN1CCc2ccccc2C1</smiles>

51
Scheme 14

\section{6 其他}

\section{1 有机光开关纳米线}

静电纺丝是将高分子流体静电雾化后分裂成聚合 物微小射流并最终固化成纤维. 通过电喷雾方法可以得 有机纳米线, 操作技术简单, 而且可以实现功能多种多 样. 我们还可以通过控制特殊结构的毛细管或者调整合 适的过程参数, 还能够控制沉积物和纳米线的方向, 实 现微制造表面的集成和超结构. Benedetto 等 ${ }^{[54]}$ 将 13 与 聚甲基丙烯酸甲酯(PMMA)混合, 通过静电纺丝方法得 到了直径为 $50 \sim 400 \mathrm{~nm}$, 高度约为 $100 \mathrm{~nm}$ 的光开关有 机纳米线. 这些有机光开关纳米线保留了螺吡喃的光异 构化性质(Eq. 14), 在紫外光照前后, 聚合物中螺吡喃分 子结构的变化导致聚合物表面亲疏水性的变化. 因为不 需要纳米光刻和对智能表面的增强控制等技术, 静电纺 丝得到的纳米纤维就可以提供可控的功能器件, 且分辨 率可以达到亚微米甚至几十纳米. 通过静电纺丝得到的 纳米纤维具有良好的稳定性和循环性能, 为下一步在纳
米尺度上的传感，芯片集成等应用研究提供了新的方 法.<smiles>CN1c2ccccc2C(C)(C)C12C=Cc1cc([N+](=O)[O-])ccc1O2</smiles>

\section{2 有机光开关 Langmuir 薄膜}

Langmuir 薄膜是一种有机单分子层薄膜, 与其他薄 膜技术相比, 其可以在几个埃米的范围内精确控制厚度 和实现精细的分子组装的特点, 显得更加突出. 而滞后 现象在材料的磁学性质, 晶体的光谱性质, 固体表面的 接触角和异构体的吸收等现象中广泛存. 而聚合物在交 变应力作用下，链段通过热运动达到新平衡需要时间， 由此引起应变落后于应力的滞后现象. 从开关和记忆器 件的角度来说, 与双稳态和相变有关的滞后现象引起了 更多的关注. Chen 等 ${ }^{[5]}$ 用十二烷基磺酸钠(SDS) 将螺吡 喃分子包覆在其胶束内，通过后扩散的方法制备了含螺 吡喃的 SDS 胶束溶液的聚烯丙基胺一葡聚糖微凝胶/透明 质酸钠 $(\mathrm{PAH}-\mathrm{D} / \mathrm{HA})$ 薄膜. 他们研究了此薄膜中螺吡喃 分子的光致变色性质，为制备新型的光致变色薄膜提供 了新的方法. Matsumoto 等 ${ }^{[56]}$ 在等压条件下, 在光响应 的 Langmuir 薄膜中第一次发现了热滞后现象, 而并未 检测出化合物 52 (Eq. 15)的结构变化, 故 Langmuir 薄膜 中的这一滞后现象应该与发生在 Langmuir 薄膜中的相 变有关. 利用螺吡喃分子在光罩薄膜中形成了规整的 J聚集态, 他们得到很好的线状条纹.

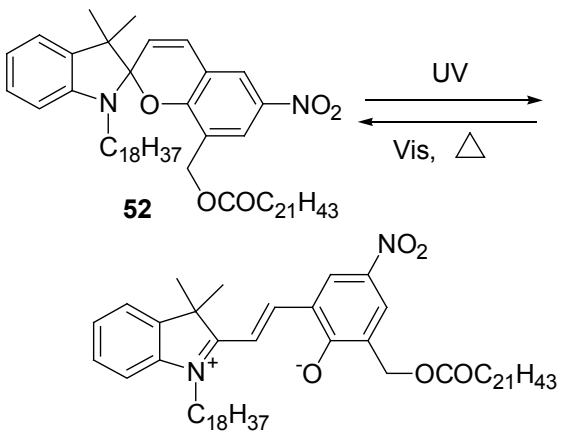

\section{3 水溶性螺吡喃分子}

Kohl-Landgraf 等 ${ }^{[57]}$ 合成了含哌啶单元的螺吡喃衍 生物 53, 并将其季铵盐化得到了水溶性的螺吡喃分子 54 (Scheme 15). 他们利用飞秒可见洜浦/可见和红外探 测光谱研究了水溶液中化合物 $\mathbf{5 4}$ 的可逆开关性质, 揭 
示了螺吡喃的开环速度明显快于部花菁分子的闭环速 度. 螺吡喃的苯并吡喃部分他们选择了吡啶，没有硝基 强拉电子基团的影响, 使螺吡喃分子更趋于稳定. 相比 于其他硝基螺吡喃分子, 衍生物 54 具有更好的水溶性 和稳定性以及快速有效的开关性质, 使 54 可能成为生 物应用领域更具有吸引力的工具.
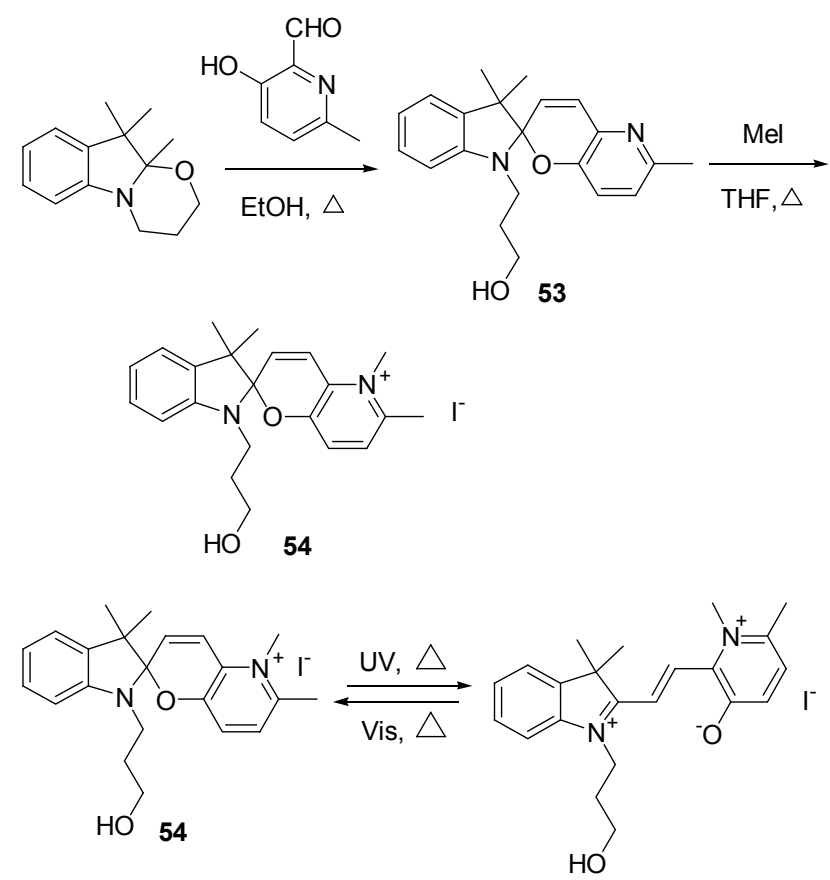

Scheme 15

\section{7 结论与展望}

光致变色化合物以其特殊的光化学和光物理特性, 引起了大家的广泛关注. 进一步的研究证明, 光致变色 化合物在化学、物理学、材料学、生物学或相关的交叉 学科等领域多发挥着积极的作用, 得到了广泛的研究和 应用, 如超高密度信息存储, 光电子器件, 化学以及生 物传感和成像, 特别是最近出现的超分辨苂光显微镜细 胞成像技术.

受衍射极限的影响, 传统的荧光显微镜的分辨率最 高只能达到 $200 \mathrm{~nm}$, 而在远场苂光显微学中超分辨成 像技术发展使我们可以在纳米尺度观察细胞的活动. 利 用不同激发波长的光刺激分子光开关而实现其荧光的 “开”和“关”的性质，使其可以作为新型的荧光探针，用 于细胞成像, 特别是细胞中微结构的超分辨成像. 而将 螺吡喃分子通过聚合后得到的光开关纳米粒子, 其荧光 量子产率和抗疲劳性质以及抗漂白性质均有大幅度改 善, 为其在生物成像领域的应用奠定了良好的基础. 进 一步探索发展研究合成新型的抗疲劳性质优良同时具 有高荧光量子产率的有机光开关分子应用于细胞标记
和超分辨成像将具有更加诱人的前景.

\section{References}

[1] Irie, M. Chem. Rev. 2000, 100, 1685.

[2] (a) Raymo, F. M.; Tomasulo, M. Chem. Soc. Rev. 2005, 34, 327. (b) Yildiz, I.; Deniz, E.; Raymo, F. M. Chem. Soc. Rev. 2009, 38, 1859.

(c) Tian, H.; Yang, S. Chem. Soc. Rev. 2004, 33, 85.

(d)Yang S.-H.; Pang M.-L.; Meng J.-B. Chin. J. Org. Chem. 2011, 31, 1725 (in Chinese). (杨素华, 庞美丽, 孟继本, 有机化学, 2011, 31, 1725.)

[3] Zacharias, P.; Gather, M. C.; Köhnen, A.; Rehmann, N.; Meerholz, K. Angew. Chem., Int. Ed. 2009, $48,4038$.

[4] (a) Kawata, S.; Kawata, Y. Chem. Rev. 2000, 100, 1777. (b) Tian, H. Angew. Chem., Int. Ed. 2010, 49, 4710.

[5] (a) Raymo, F. M.; Giordani, S. Org. Lett. 2001, 3, 1833. (b) Raymo, F. M.; Alvarado, R. J.; Giordani, S.; Cejas, M. A. J. Am. Chem. Soc. 2003, 125, 2361 .

(c) Raymo, F. M.; Giordani, S. J. Am. Chem. Soc. 2001, 123, 4651.

[6] Inouye, M.; Ueno, M.; Kitao, T.; Tsuchiya, K. J. Am. Chem. Soc. 1990, $112,8977$.

[7] Zhang, H.; Kou, X.-X.; Zhang, Q.; Qu, D.-H.; Tian, H. Org Biomol. Chem. 2011, 9, 4051.

[8] (a) Tong, R.; Hemmati, H. D.; Langer, R.; Kohane, D. S. J. Am. Chem. Soc. 2012, 134, 8848.

(b) Achilleos, D. S.; Hatton, T. A.; Vamvakaki, M. J. Am. Chem. Soc. 2012, 134, 5726.

[9] (a) Hu, D.; Tian, Z.; Wu, W.; Wan, W.; Li, A. D. Q. J. Am. Chem. Soc. 2008, 130, 15279.

(b) Deniz, E.; Tomasulo, M.; Cusido, J.; Yildiz, I.; Petriella, M.; Bossi, M. L.; Sortino, S.; Raymo, F. M. J. Phys. Chem. C 2012, 116, 6058 .

[10] Natansohn, A.; Rochon, P. Chem. Rev. 2002, 102 , 4139.

[11] (a) Tian, H.; Wang, S. Chem. Commun. 2007, 781.

(b) Feng, Y.; Yan, Y.; Wang, S.; Zhu, W.; Qian, S.; Tian, H. J. Mater. Chem. 2006, 16, 3685.

(c) Tian, H.; Feng, Y. J. Mater. Chem. 2008, 18, 1617.

[12] Berkovic, G.; Krongauz, V.; Weiss, V. Chem. Rev. 2000, 100, 1741.

[13] (a) Donnert, G.; Keller, J.; Medda, R.; Andrei, M. A.; Rizzoli, S. O.; Lührmann, R.; Jahn, R.; Eggeling, C.; Hell, S. W. Proc. Natl. Acad. Sci. U. S. A. 2006, 103, 11440.

(b) Patterson, G. H.; Lippincott-Schwartz, J. Science 2002, 297, 1873.

[14] (a) Subach, F. V.; Patterson, G. H.; Manley, S.; Gillette, J. M.; Lippincott-Schwartz, J.; Verkhusha, V. V. Nat. Meth. 2009, 6 , 153. (b) Gurskaya, N. G.; Verkhusha, V. V.; Shcheglov, A. S.; Staroverov, D. B.; Chepurnykh, T. V.; Fradkov, A. F.; Lukyanov, S.; Lukyanov, K. A. Nat. Biotech. 2006, 24, 461.

[15] Wiedenmann, J.; Ivanchenko, S.; Oswald, F.; Schmitt, F.; Röcker, C.; Salih, A.; Spindler, K.-D.; Nienhaus, G. U. Proc. Natl. Acad. Sci. U. S. A. 2004, 101, 15905.

[16] Ando, R.; Mizuno, H.; Miyawaki, A. Science 2004, 306, 1370.

[17] (a) Dempsey, G. T.; Bates, M.; Kowtoniuk, W. E.; Liu, D. R.; Tsien, R. Y.; Zhuang, X. J. Am. Chem. Soc. 2009, 131, 18192.

(b) Zhuang, X. Nat. Photon. 2009, 3, 365.

(c) Huang, B.; Wang, W.; Bates, M.; Zhuang, X. Science 2008, 319 , 810 .

[18] Davis, D. A.; Hamilton, A.; Yang, J.; Cremar, L. D.; Van Gough, D.; Potisek, S. L.; Ong, M. T.; Braun, P. V.; Martinez, T. J.; White, S. R. Moore, J. S.; Sottos, N. R. Nature 2009, 459, 68.

[19] O'Bryan, G.; Wong, B. M.; McElhanon, J. R. ACS Appl. Mater. 
Interfaces 2010, 2, 1594.

[20] Lee, C. K.; Davis, D. A.; White, S. R.; Moore, J. S.; Sottos, N. R.; Braun, P. V. J. Am. Chem. Soc. 2010, 132, 16107.

[21] (a) Kim, H.-S.; Kim, Y.-H.; Kim, T.-H.; Noh, Y.-Y.; Pyo, S.; Yi, M. H.; Kim, D.-Y.; Kwon, S.-K. Chem. Mater. 2007, 19, 3561.

(b) Orgiu, E.; Masillamani, A. M.; Vogel, J.-O.; Treossi, E.; Kiersnowski, A.; Kastler, M.; Pisula, W.; Dotz, F.; Palermo, V.; Samori, P. Chem. Commun. 2012, 48, 1562.

[22] Li, Y.; Zou, Y. Adv. Mater. 2008, 20 (15), 2952.

[23] Harpham, M. R.; Süzer, Ö.; Ma, C.-Q.; Bäuerle, P.; Goodson, T. J. Am. Chem. Soc. 2009, 131, 973.

[24] (a) Camurlu, P.; Cirpan, A.; Toppare, L. J. Electroanal. Chem. 2004, $572,61$.

(b) Mortimer, R. J. Electrochim. Acta 1999, 44, 2971.

(c) Sahin, E.; Camurlu, P.; Toppare, L.; Mercore, V. M.; Cianga, I.; Yağc1, Y. J. Electroanal. Chem. 2005, 579, 189.

[25] Wagner, K.; Byrne, R.; Zanoni, M.; Gambhir, S.; Dennany, L.; Breukers, R.; Higgins, M.; Wagner, P.; Diamond, D.; Wallace, G. G.; Officer, D. L. J. Am. Chem. Soc. 2011, 133, 5453.

[26] Bahr, J. L.; Kodis, G.; de la Garza, L.; Lin, S.; Moore, A. L.; Moore, T. A.; Gust, D. J. Am. Chem. Soc. 2001, 123, 7124.

[27] Tomasulo, M.; Kaanumal, S. L.; Sortino, S.; Raymo, F. M. J. Org. Chem. 2006, 72, 595.

[28] Karolin, J.; Johansson, L. B. A.; Strandberg, L.; Ny, T. J. Am. Chem. Soc. 1994, 116, 7801.

[29] Tomasulo, M.; Deniz, E.; Alvarado, R. J.; Raymo, F. M. J. Phys. Chem. C 2008, $112,8038$.

[30] Zhu, L.; Zhu, M.-Q.; Hurst, J. K.; Li, A. D. Q. J. Am. Chem. Soc. 2005, $127,8968$.

[31] (a) Baillet, G.; Guglielmetti, R.; Giusti, G. Mol. Cryst. Liq. Cryst. Sci. Technol., Sect. A 1994, 246 (1), 287.

(b) Campredon, M.; Luccioni-Houze, B.; Giusti, G.; Lauricella, R.; Alberti, A.; Macciantelli, D. J. Chem. Soc., Perkin Trans. 2 1997, 2559.

[32] Inouye, M.; Akamatsu, K.; Nakazumi, H. J. Am. Chem. Soc. 1997, $119,9160$.

[33] Xu, Z.; Baek, K.-H.; Kim, H. N.; Cui, J.; Qian, X.; Spring, D. R.; Shin, I.; Yoon, J. J. Am. Chem. Soc. 2009, 132, 601.

[34] Shao, N.; Wang, H.; Gao, X.; Yang, R.; Chan, W. Anal. Chem. 2010, 82,4628 .

[35] Shao, N.; Jin, J.; Wang, H.; Zheng, J.; Yang, R.; Chan, W.; Abliz, Z. J. Am. Chem. Soc. 2009, 132, 725.

[36] Champagne, B.; Plaquet, A.; Pozzo, J.-L.; Rodriguez, V.; Castet, F. J. Am. Chem. Soc. 2012, 134, 8101.

[37] (a) Hirano, T.; Kikuchi, K.; Urano, Y.; Higuchi, T.; Nagano, T. J. Am. Chem. Soc. 2000, 122, 12399.

(b) Maruyama, S.; Kikuchi, K.; Hirano, T.; Urano, Y.; Nagano, T.
J. Am. Chem. Soc. 2002, 124, 10650.

(c) Hirano, T.; Kikuchi, K.; Urano, Y.; Nagano, T. J. Am. Chem. Soc. 2002, 124, 6555.

[38] Guo, X.; Zhang, D.; Zhou, Y.; Zhu, D. J. Org. Chem. 2003, 68, 5681.

[39] (a) Wurthner, F.; Stolte, M. Chem. Commun. 2011, 47, 5109.

(b) Huang, C.; Barlow, S.; Marder, S. R. J. Org. Chem. 2011, 76, 2386.

(c) Li, C.; Wonneberger, H. Adv. Mater. 2012, 24, 613.

[40] Guo, X.; Zhang, D.; Zhu, D. Adv. Mater. 2004, 16, 125.

[41] Saita, S.; Yamaguchi, T.; Kawai, T.; Irie, M. ChemPhysChem 2005, 6,2300 .

[42] Denk, W.; Strickler, J.; Webb, W. Science 1990, 248, 73.

[43] So, P. T. C.; Dong, C. Y.; Masters, B. R.; Berland, K. M. Annu. Rev. Biomed. Eng. 2000, 2, 399.

[44] Harada, J.; Nakajima, R.; Ogawa, K. J. Am. Chem. Soc. 2008, 130, 7085.

[45] Yuan, W.; Sun, L.; Tang, H.; Wen, Y.; Jiang, G.; Huang, W.; Jiang, L.; Song, Y.; Tian, H.; Zhu, D. Adv. Mater. 2005, 17, 156.

[46] Zhu, L.; Wu, W.; Zhu, M.-Q.; Han, J. J.; Hurst, J. K.; Li, A. D. Q. J. Am. Chem. Soc. 2007, 129, 3524.

[47] Tian, Z.; Wu, W.; Wan, W.; Li, A. D. Q. J. Am. Chem. Soc. 2009, 131,4245 .

[48] Tian, Z.; Li, A. D. Q.; Hu, D. Chem. Commun. 2011, 47, 1258.

[49] Zhu, M.-Q.; Zhu, L.; Han, J. J.; Wu, W.; Hurst, J. K.; Li, A. D. Q. J. Am. Chem. Soc. 2006, 128, 4303.

[50] Williams, R. M.; Zipfel, W. R.; Webb, W. W. Curr. Opin. Chem. Biol. 2001, 5, 603 .

[51] Zhu, M.-Q.; Zhang, G.-F.; Li, C.; Aldred, M. P.; Chang, E.; Drezek, R. A.; Li, A. D. Q. J. Am. Chem. Soc. 2011, 133, 365.

[52] Tan, C.-C.; Zhao, Z.-L.; Gao, J.; Lei, J.-X. Acta Chim. Sinica 2012, 70, 1095 (in Chinese). (谭春斌, 赵泽琳, 高峻, 雷景新, 化学学报, 2012, 70, 1095.)

[53] Pang, M.-L.; Yang, T.-T.; Li, J.-J.; Yang, S.-H.; Lou, Z.-G.; Han, J.; Meng, J.-B. Acta Chim. Sinica 2010, 68, 1895 (in Chinese). (庞美丽, 杨涛涛, 李晶晶, 杨素华, 娄志刚, 韩杰, 孟继本, 化 学学报, 2010, 68, 1895.)

[54] Benedetto, F. D.; Mele, E.; Camposeo, A.; Athanassiou, A.; Cingolani, R.; Pisignano, D. Adv. Mater. 2008, $20,314$.

[55] Chen D.-D.; Wang L.; Sun J.-Q. Acta Chim. Sinica 2012, 70, 1779 (in Chinese). (陈栋栋, 王林, 孙俊奇, 化学学报, 2012, 70, 1779.)

[56] Matsumoto, M.; Nakazawa, T.; Mallia, V. A.; Tamaoki, N.; Azumi, R.; Sakai, H.; Abe, M. J. Am. Chem. Soc. 2004, 126, 1006.

[57] Kohl-Landgraf, J.; Braun, M.; Özçoban, C.; Gonçalves, D. P. N.; Heckel, A.; Wachtveitl, J. J. Am. Chem. Soc. 2012, 134, 14070. 